\title{
1 Gut Microbiome of Helminth Infected Indigenous Malaysians Is Context Dependent
}

3 Mian Zi Tee ${ }^{1}$, Yi Xian Er², Alice V. Easton ${ }^{3}$, Nan Jiun Yap ${ }^{2}$, Ii Li Lee ${ }^{4}$, Joseph Devlin ${ }^{3}$, Ze Chen ${ }^{3}$,

4 Kee Seong Ng ${ }^{5}$, Poorani Subramanian ${ }^{6}$, Angelina Angelova ${ }^{6}$, Shushan Sargsian $^{3,7}$, Romano Ngui ${ }^{2}$,

5 Christopher Chiong Meng Boey ${ }^{8}$, Kek Heng Chua ${ }^{1}$, Ken Cadwell ${ }^{3,7,9}$, Yvonne Ai Lian Lim²*, P’ng

$6 \quad$ Loke $^{10^{*}}$, Soo Ching Lee ${ }^{10^{*}}$

$8{ }^{1}$ Department of Biomedical Science, Faculty of Medicine, Universiti Malaya, Kuala Lumpur,

9 Malaysia.

$10{ }^{2}$ Department of Parasitology, Faculty of Medicine, Universiti Malaya, Kuala Lumpur, Malaysia.

$11{ }^{3}$ Department of Microbiology, New York University Grossman School of Medicine, New York, NY,

12 United States.

$13{ }^{4}$ Kulliyyah of Medicine and Health Sciences, University Islam Antarabangsa Sultan Abdul Halim

14 Mu'adzam Shah, 09300 Kuala Ketil, Kedah.

$15{ }^{5}$ Department of Gastroenterology, Faculty of Medicine, Universiti Malaya, Kuala Lumpur,

16 Malaysia.

$17{ }^{6}$ Bioinformatics and Computational Biosciences Branch, Office of Cyber Infrastructure and

18 Computational Biology, National Institute of Allergy and Infectious Diseases, National Institutes

19 of Health, Bethesda, MD, USA.

$20 \quad{ }^{7}$ Kimmel Center for Biology and Medicine at the Skirball Institute, New York University Grossman

21 School of Medicine, New York, NY, United States.

$22{ }^{8}$ Department of Paediatrics, Faculty of Medicine, Universiti Malaya, Kuala Lumpur, Malaysia 
$23{ }^{9}$ Division of Gastroenterology, Department of Medicine, New York University Langone Health,

24 New York, NY, United States.

$25{ }^{10}$ Type 2 Immunity Section, Laboratory of Parasitic Diseases, National Institute of Allergy and 26 Infectious Diseases, National Institute of Health, Bethesda, MD, United States.

28 Mian Zi Tee and Yi Xian Er contributed equally to this study.

$30 *$ Corresponding Authors:

31 Yvonne Ai Lian Lim: limailian@um.edu.my;

32 P’ng Loke: png.loke@nih.gov;

33 Soo Ching Lee: sooching.lee@nih.gov

35 Running title: Microbiome of Indigenous Malaysians with Helminths

37 Key Words: helminth, microbiome, metagenomic sequencing, indigenous population,

38 albendazole

\section{Abstract}

41 While microbiomes in industrialized societies are well characterized, indigenous populations with

42 traditional lifestyles have microbiomes that are more akin to those of ancient humans. However,

43 metagenomic data in these populations remains scarce and the association with soil-transmitted

44 helminth infection status is unclear. Here, we sequenced 650 metagenomes of indigenous

45 Malaysians from 5 villages with different prevalence of helminth infections. Individuals from 
46 villages with higher prevalence of helminth infections have more unmapped reads and greater

47 microbial diversity. Microbial community diversity and composition were most strongly

48 associated with different villages and the effects of helminth infection status on the microbiome

49 varies by village. Longitudinal changes in the microbiome in response to albendazole anthelmintic

50 treatment was observed in both helminth infected and uninfected individuals. Inference of bacterial

51 population replication rates from origin of replication analysis identified specific replicating taxa

52 associated with helminth infection. Our results indicated that helminth effects on the microbiota

53 was highly dependent on context and effects of albendazole on the microbiota can be confounding

54 for the interpretation of deworming studies. Furthermore, a substantial quantity of the microbiome

55 remains undescribed and this large dataset from indigenous populations associated with helminth

56 infections should facilitate characterization of the disappearing microbiome from developed

57 industrialized societies.

59 Introduction

60 Industrialization is associated with reduced diversity of the microbiome in the human population

61 [1], which could influence a range of physiological processes including nutrition, metabolism,

62 immunity, neurochemistry and drug metabolism [2]. Traditional indigenous populations have

63 substantially greater microbial diversity than individuals living in industrialized societies.

64 Nonetheless, our current knowledge of the human gut microbiome [3] is overrepresented by data

65 available from industrialized countries and does not fully address the under-sampling of

66 indigenous populations, which may contain the largest source of disappearing microbes that could

67 be utilized for restoration of the ancestral human microbiome [4] . 
Throughout evolution, helminths have coexisted with the gut microbiota in their mutual

69 host [5] and the reduced prevalence of helminth infections from modern practices could contribute

70 to the "hygiene hypothesis" [6]. By co-evolution, helminths and microbiota are likely to interact

71 and affect each other, thus having significant effects on the host. While the effect of helminth

72 colonisation on the human gut microbiota has been increasingly studied, the results reported have

73 been inconsistent. Some studies find that helminth colonization changes gut microbial diversity

74 and composition and/or a shift in abundance of certain bacterial taxa [7-13], while others show no

75 apparent changes in gut microbial profiles $[14,15]$. These divergent conclusions could be

76 attributed to different confounders from different geographical locations (e.g. Malaysia [7, 13],

77 Indonesia [10], Liberia [10], Tanzania [12], Western Kenya [11] and Ecuador [14]), different

78 prevalence of helminth species (e.g. Trichuris sp. [14], hookworm [15], Ascaris sp. [11],

79 Strongyloides sp. [9] and Schistosoma spp. [16]), as well as different approaches taken (natural or

80 experimental infection, types of sequencing method and analysis approaches). Additionally, the

81 direct impact of anthelmintic treatment on the gut microbiome is unclear. While some studies

82 found differences following deworming treatment [11, 13], others have found no impact of

83 treatment on gut microbiota profiles [14]. Other studies that examined anthelmintic albendazole

84 effects on the gut microbiota utilize primarily $16 \mathrm{~S}$ rRNA sequencing $[10,11,14,17,18]$. Hence

85 a large study incorporating metagenomic sequencing with helminth infection status, albendazole

86 treatment and additional control groups may provide greater insights into these complex

87 interactions.

88 Most studies above utilized $16 \mathrm{~S}$ rRNA sequencing to characterize the gut microbiota.

89 Shotgun metagenomic approaches enable higher taxonomic resolution at the species level, and can

90 identify not only bacteria, but also archaea, fungi and viruses [19, 20]. However, the deficiency of 
91 reference databases for mapping metagenomic data can make it a challenge to profile

92 uncharacterized microorganisms. Recently, an approach of assembling sequencing reads into

93 contigs and binning them into putative genomes, known as metagenome assembled genomes

94 (MAGs), has enabled retrieving genomes directly from samples without the need of culturing

95 organisms [21, 22]. The Unified Human Gastrointestinal Genome (UHGG) established an

96 integrated catalog of prokaryotic genomes containing 204,938 non-redundant genomes that

97 represent 4,644 prokaryotic species [3] by combining recent studies with large scale assembly of

98 MAGs from human microbiome data [22-24] as well as two culture-based studies that sequenced

99 genomes from cultivated human gut bacteria [25, 26]. The Human Reference Gut Microbiome

100 (HRGM) catalog expanded on UHGG to include under-represented Asian metagenomes from

101 Korea, India and Japan [27] and added 780 new species from the newly assembled genomes [27].

102 However, Southeast-Asian countries remain under-represented.

103 In this study, we utilized shotgun metagenomics on 650 Malaysian stool samples to

104 investigate helminth-gut microbiome interactions by both cross-sectional and longitudinal

105 analyses. The large sample size allowed us to examine these interactions in 5 different villages

106 from different locations and lifestyle. Examination of anthelmintic treated uninfected individuals

107 in the longitudinal phase enabled assessment of albendazole effects on the gut microbiome.

108 Metagenomic data enabled investigating the replication rates of individual bacterial species under

109 different conditions. Since a substantial quantity of the microbiome remains undescribed, this large

110 dataset from indigenous populations with traditional lifestyles from the under-represented South

111 East Asian region provides new insights into helminth-gut microbiome interactions and more

112 comprehensive metagenomic sequences for future human gut microbiome studies. 


\section{Results}

\section{Gut microbiome analysis of indigenous Malaysians and urban controls}

116 To identify and characterize helminth-associated microbiome effects, this study consisted of a

117 cross-sectional component that compares urban individuals $(\mathrm{N}=56)$ living in Kuala Lumpur (KL)

118 with indigenous Orang Asli (OA) (N=351) from five different villages (Figures S1 \& S2), as well

119 as a longitudinal component to examine changes to the microbiome after anthelminthic

120 (albendazole) treatment. A total of 650 fecal samples (including longitudinal samples) were

121 analyzed by metagenomic sequencing generating $11,480,206,516$ paired reads after quality control

122 and contamination removal (Figure S3). We compared different OA villages, which have different

123 prevalence of soil-transmitted helminth infections (Figures S1 \& S2). In the longitudinal phase of

124 the study, consented OA subjects were treated with 400mg albendazole for 3 consecutive days

125 after collection of the first fecal sample. At 21 - and 42-days following treatment, additional fecal

126 samples were collected, however this phase of the study was disrupted by the COVID-19

127 pandemic, reducing the number of paired samples available. KL subjects were not treated with

128 albendazole and provided only one sample. Questionnaire data were collected and analyzed for

129 some of the study subjects $(n=340)$.

130 When we first mapped the metagenomic sequences to RefSeq (i.e., bacteria, protozoa,

131 fungi, viral, archaea genomes), we observed a very low percentage of mapped reads (median:

$13241.6 \%$ ). However, when we mapped the sequences to databases that incorporate metagenome

133 assembled genomes (i.e., HRGM [27] and UHGG [3], the percentage of sequencing reads mapped

134 to HRGM (median: 91.5\%) and UHGG (median: 87.9\%) was much higher than RefSeq (Figure

135 1A). Additionally, the percentage of mapped reads to all three databases was higher in KL subjects 
136 than the OA population [HRGM: $\mathrm{p}=2.6 \mathrm{e}^{-11}$; UHGG: $\mathrm{p}=1.2 \mathrm{e}^{-07}$; RefSeq: $\mathrm{p}=2 \mathrm{e}^{-07}$ ] (Figure 1A),

137 indicating that there are more unknown microbial genomes in the OA population.

138 Utilizing HRGM, we determined the core microbiota for the Malaysian population and

139 found that 237 core bacterial species were 100\% shared among the subjects (Figure 1B;

140 Supplementary Figures S4A-E). The most abundant phylum was Firmicutes A, the majority of

141 which were uncultured species [3] (Figure 1B). Agathobacter rectalis, Balutia_A wexlerae and

142 Agathobacter faecis were the main species from Firmicutes A (Figure 1B). Using a cross-validated

143 random forest model to identify core microbiota species driving the variation between OA vs KL

144 subjects, we achieved a mean prediction accuracy of 98.05\% at a kappa of 96.06\% (out-of-bag

145 error $=1.8 \%$ ). Megamonas funiformis, Phocaeicola pleneius A, Bacteroides stercoris,

146 Phocaeicola massiliensis and HRGM Genome 3145 were the top 5 predictors between OA and

147 KL subjects (Figure 1C). Of these, HRGM Genome 3145, Gemmiger sp900539695 and Blautia A

148 sp000436615 were more abundant in OA subjects, while Megamonas funiformi, Phocaeicola

149 pleneius $A$ and Bacteroides stercoris were more abundant in KL subjects (Supplementary Figure

150 S5A \& B). The bacterial species with the largest variation (cut-off 6.0 for the coefficient of

151 variation) among the core gut microbiota is shown in Figure S6A.

152 The Orang Asli live in different geographical settings and have distinctive culture and

153 lifestyle. We found that KL subjects have higher mapped reads than all OA villages (Figure 1D;

154 Supplementary Figures S6B \& C), and the percentage of mapped reads from both villages Rasau

$155\left(\mathrm{p}=2 \mathrm{e}^{-16}\right)$ and Legong $\left(\mathrm{p}=2 \mathrm{e}^{-16}\right)$ were markedly lower compared to KL (Figure 1D; Supplementary

156 Figures S6B \& C). Using a reference independent strategy to confirm our findings, we compared

157 the beta-diversity (distributions of individuals pairwise distances in reference to KL) with k-mers

15821 sketches at the genus level (Figure 1E), showing comparable results. In addition, we observed 
that Rasau and Legong had the highest beta-diversity and nucleotide dissimilarity compared to KL

160 (Figure 1E). Moreover, comparison of bacterial communities across geographical locations using

161 Jaccard distance revealed substantial differences between villages [ADONIS: $\mathrm{p}=0.001, \mathrm{R}^{2}=0.073$;

162 Analysis of similarity (ANOSIM): $\mathrm{p}=0.001, \mathrm{R}=0.215$ ] (Figure 1F, Supplementary Table S1). From

163 the Principal Coordinate Analysis (PCoA) plot (Figure 1F), we observed clustering of the samples

164 from Rasau and Legong. Conversely, the samples from Bangkong and Sepat were clustered 165 together with KL while Judah exhibited a more dispersed distribution. Hence, OA subjects in 166 Rasau and Legong were more similar in gut microbial composition, and were different from KL 167 and other villages. Equivalent beta-diversity results were observed with other k-mers sketches (31 168 and 51) and at the species level (Figures S7A-G).

171 We determined the infection intensity and the prevalence of intestinal helminth infection among 172 the 351 OA subjects and found that Trichuris infection $(61.8 \%, \mathrm{n}=217)$ was the most predominant, 173 followed by hookworm $(20.8 \%, 73)$ and Ascaris $(17.9 \%, 63)$ infections (Figure 2A). The 174 distribution of age and gender of these subjects are shown in Figures S8A \& B. The overall 175 prevalence of helminth infection was 67.2\% $(\mathrm{n}=236)$ (Figure 2A) and infection intensity was 176 summarized in Figure S8C. For beta diversity, based on PCoA, there were differences in gut 177 microbiome between infected and uninfected individuals, however, statistically the effect size was 178 small (ADONIS: $\mathrm{p}=0.001, \mathrm{R}^{2}=0.024$; ANOSIM: $\mathrm{p}=0.001, \mathrm{R}=0.145$ ) (Figure 2B, Supplementary 179 Table S1), which was also the case for Bray-curtis distance and NMDS ordination (Supplementary 180 Figure S9A-C). 
A

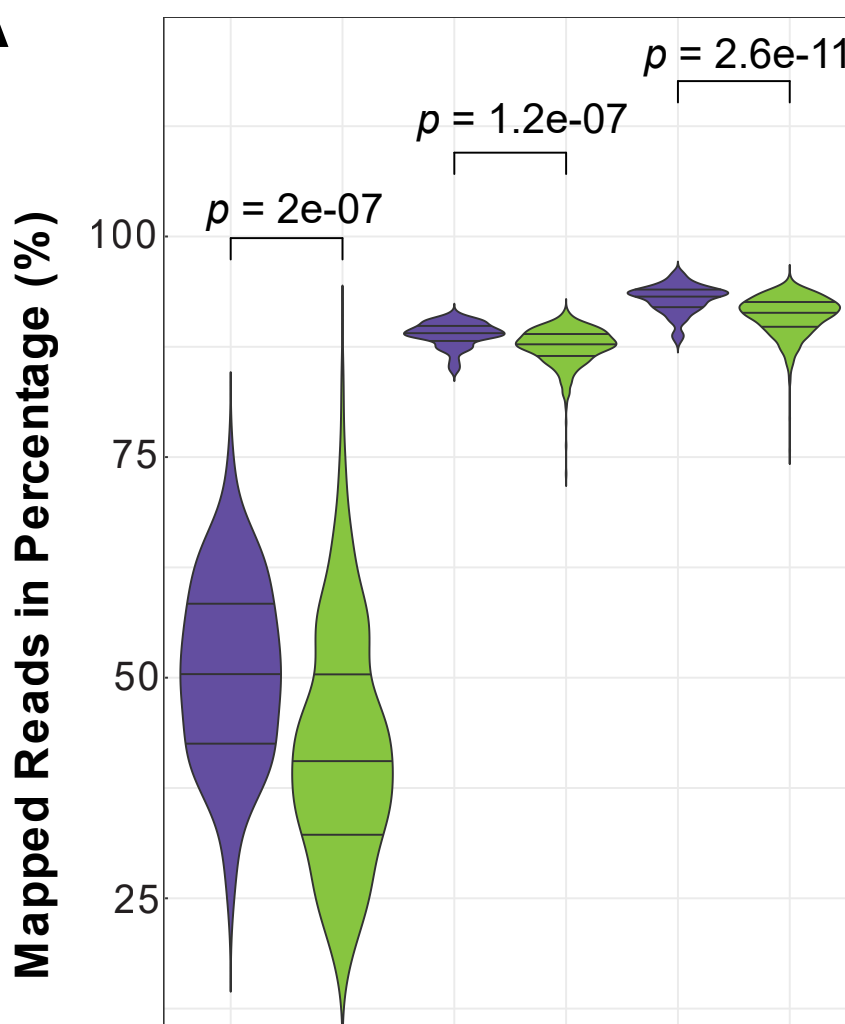

D

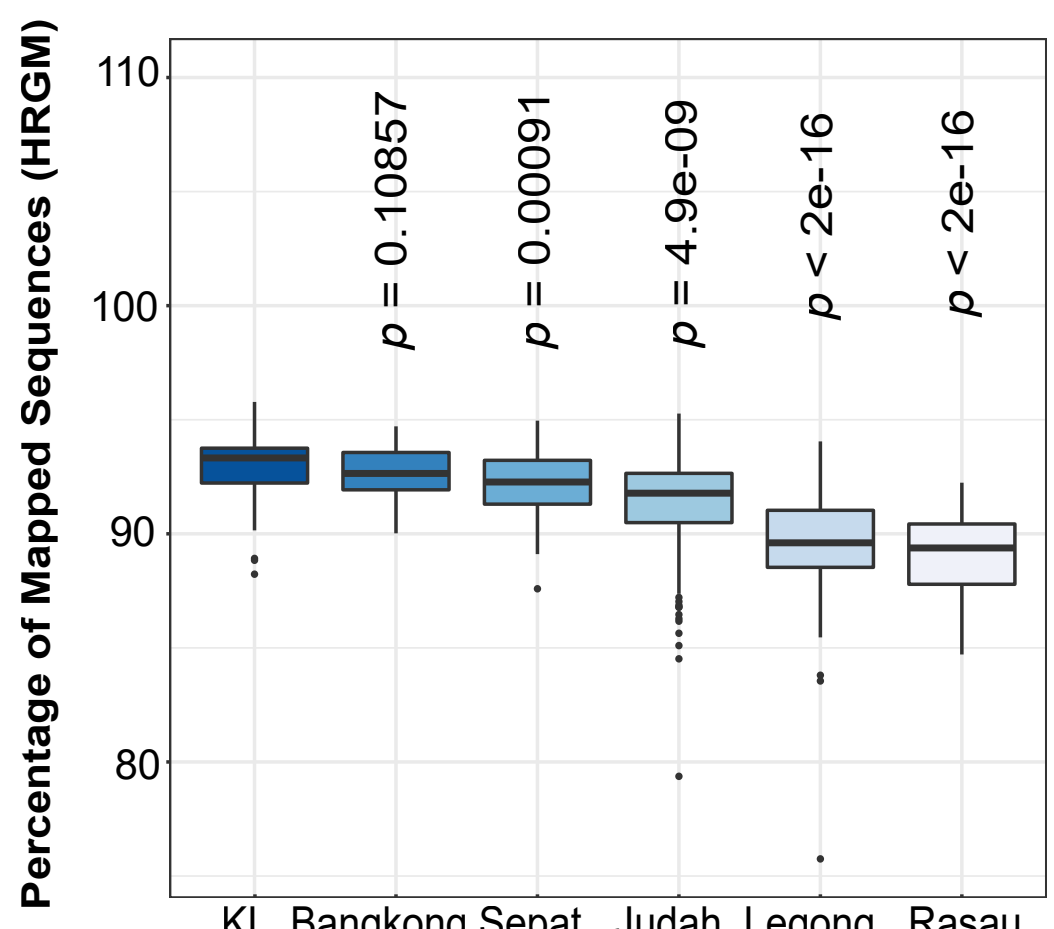

KL Bangkong Sepat Judah Legong Rasau

E

k21

bioRxiv preprint doi: https://dol.org/10.1101/2022.01.21.477162; this version posted January 24, 2022. The ceppright holder for this preprint

(which was not certified by peer review) is the author/funder. All rights reserved. No reuse allowed without permission.

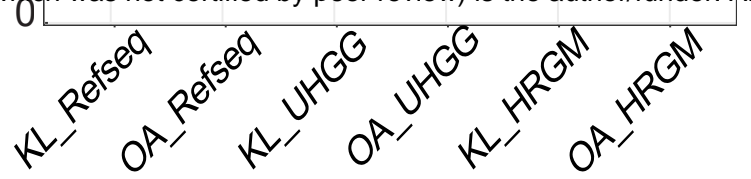

B

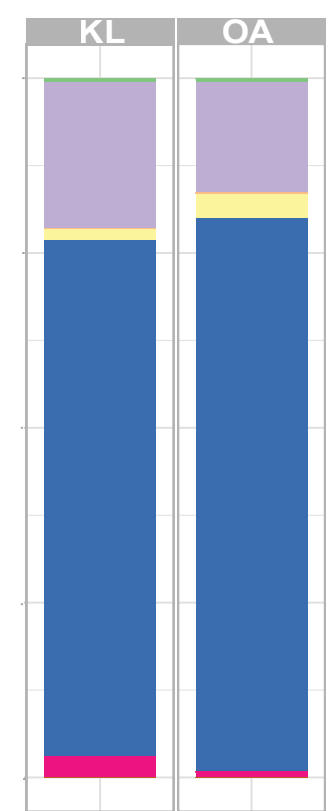

Phylum

Actinobacteriota

Bacteroidota

Cyanobacteria

Firmicutes

Firmicutes $A$

Firmicutes $C$

Proteobacteria

C

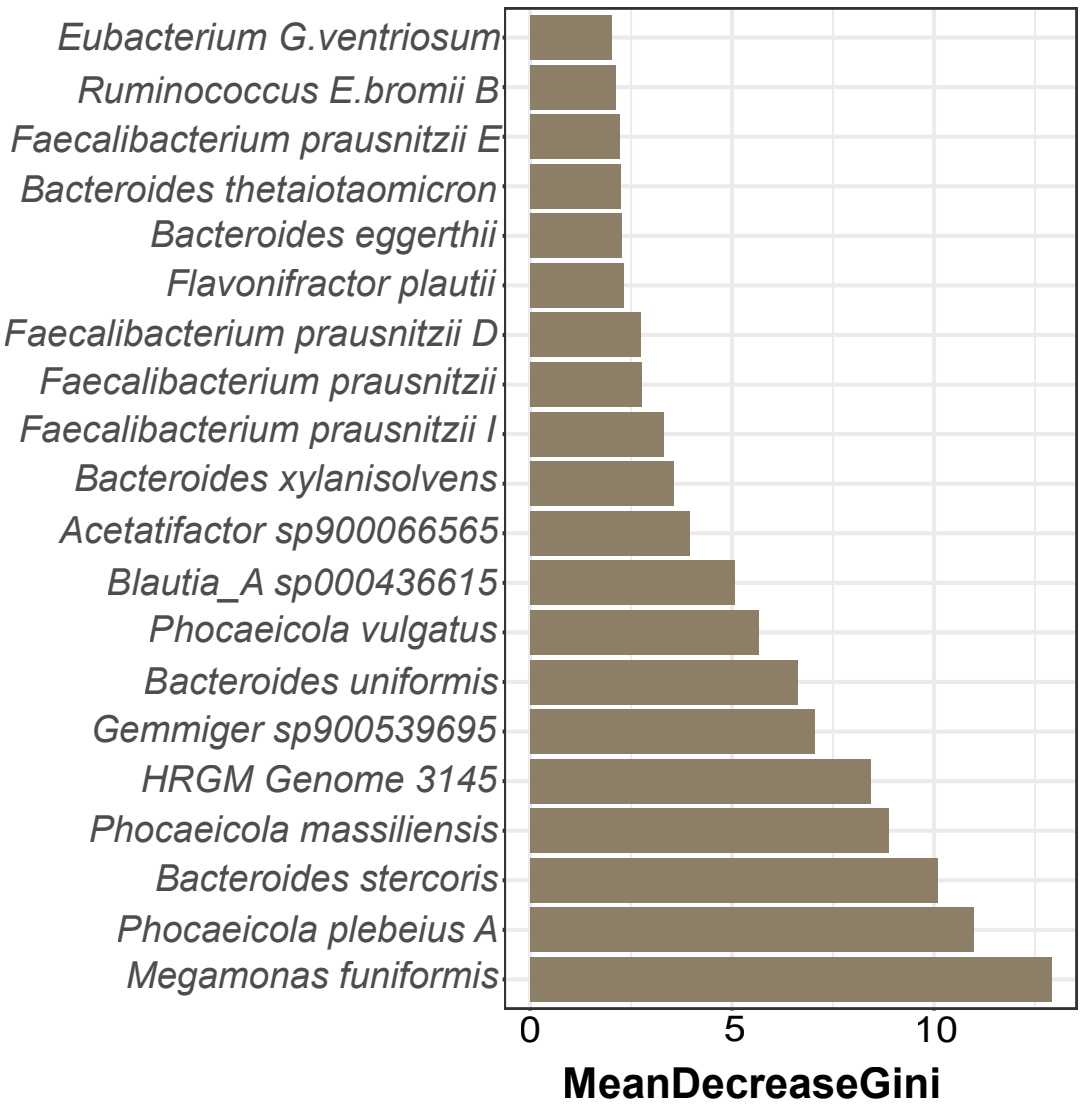

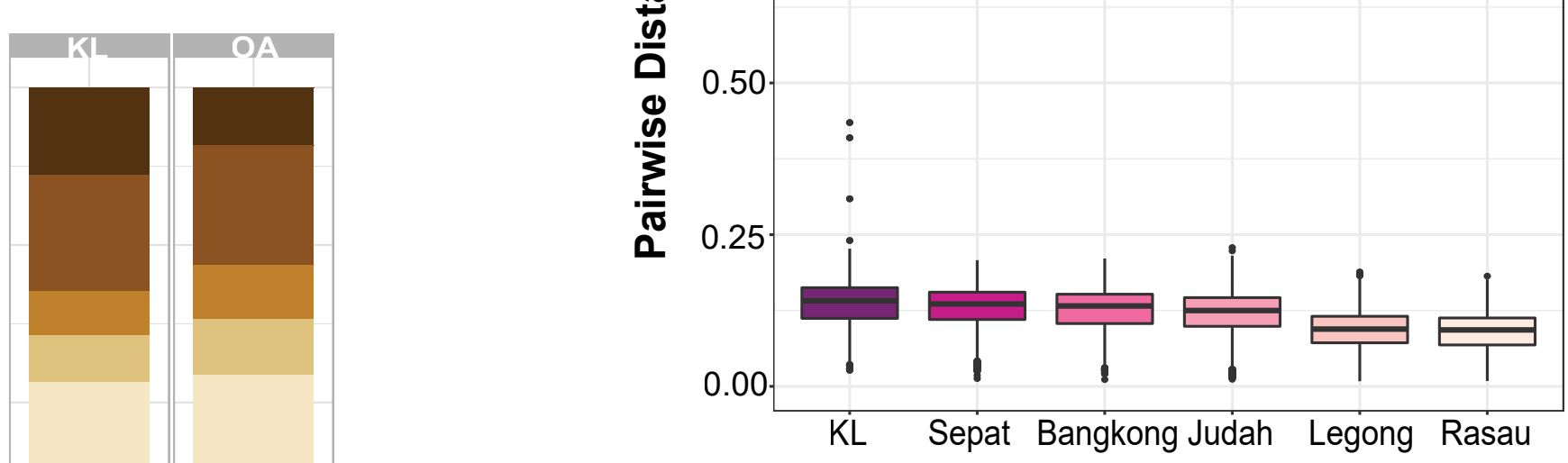

Genus_HRGM

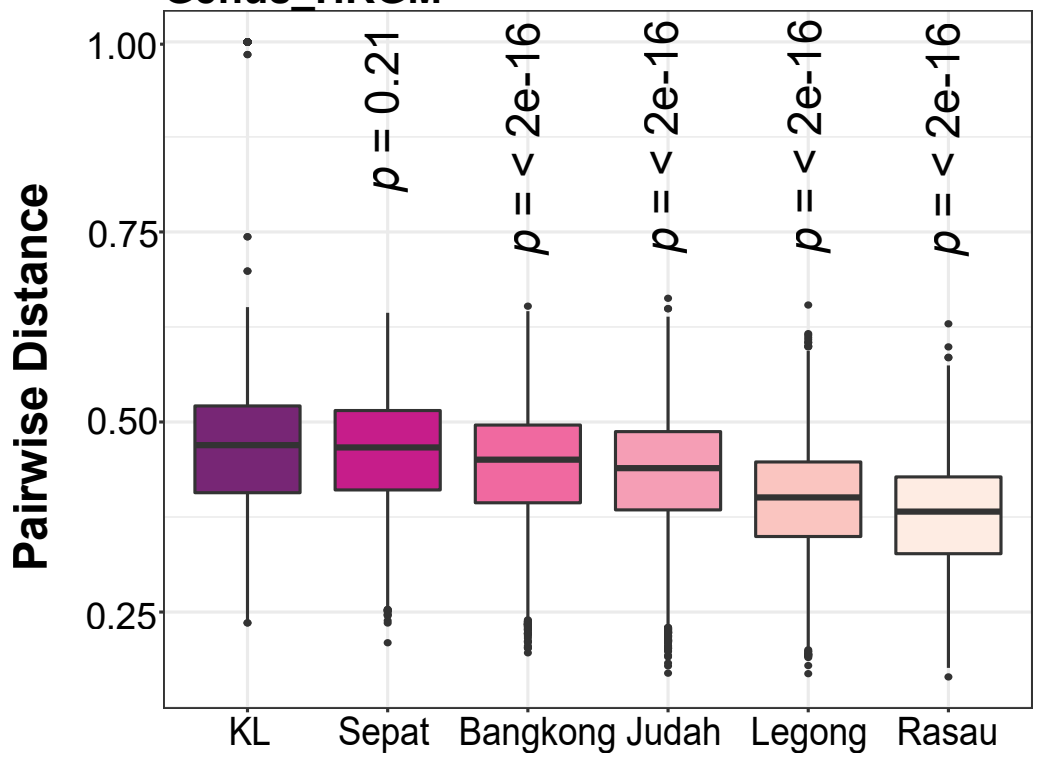

$\mathbf{F}$

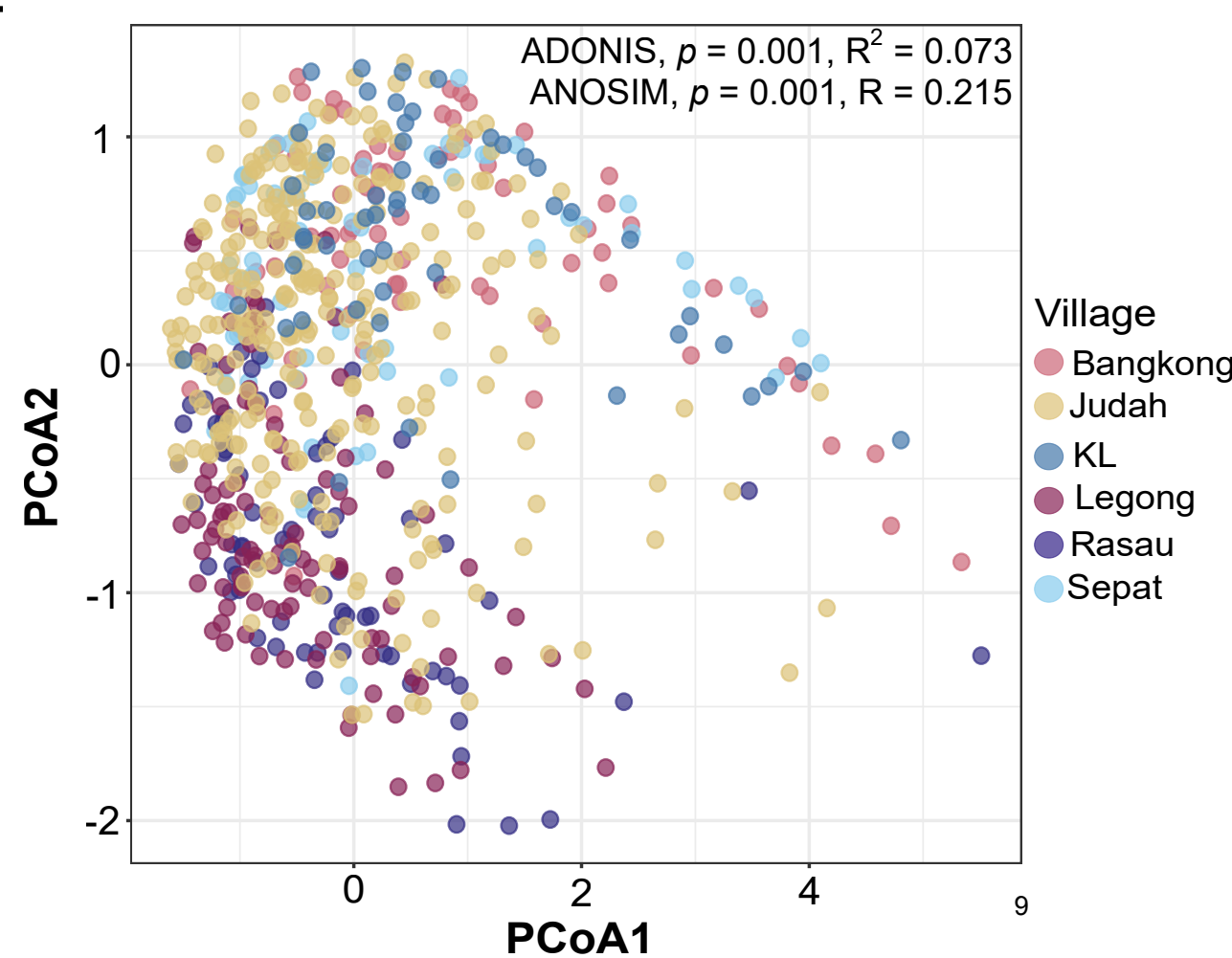


182 Fig. 1 Variation in the gut microbiome of 650 Malaysians from Orang Asli (OA) villages

183 and Kuala Lumpur (KL).

184 A Violin plots illustrating the percentage of mapped reads with RefSeq (i.e., Bacteria, protozoa,

185 fungi, viral, archaea), Unified Human Gastrointestinal Genome (UHGG) and Human Reference

186 Gut Microbiome (HRGM) databases between OA (green) and KL (purple) samples. B Relative

187 abundance of phyla from the 237 species of the core gut microbiota of OA and KL populations

188 (Left). The relative abundance of the main species from Firmicute A (Right). C Bar plot of the top

18920 species from the core gut microbiota that best predict the difference between OA and KL

190 cohorts using a Random Forest classification model. D The percentage of mapped reads to the

191 HRGM database for samples from different OA villages and KL. E Pairwise beta diversity

192 comparisons of all villages to the KL cohort, assessed by Jaccard distance based on the distance

193 of nucleotide $\mathrm{k}$-mer sketches $(\mathrm{k}=21)$, at approximately genus level (Top) and genus-level

194 classification (Bottom). F Principal Coordinates Analysis (PCoA) of Jaccard distance based on the

195 gut metagenomic profiles in all samples, with individuals from different geographical locations

196 denoted by specific color (ADONIS: $p=0.001, \mathrm{R}^{2}=0.073$; ANOSIM: $\mathrm{p}=0.001, \mathrm{R}=0.215$ ). The $\mathrm{p}-$

197 values for A, D, E is computed using Wilcoxon rank sum test. 
For alpha diversity, we observed higher species richness in the samples from infected

206 subjects $\left(\mathrm{p}=2.50 \mathrm{e}^{-5}\right)$ (Figure $\left.2 \mathrm{C}\right)$. Individuals infected with either single $(\mathrm{p}=0.005)$ or multiple

207 species of helminths ( $\mathrm{p}=0.033$ ) had higher species richness (Figure 2C). Trichuris infected OA

$208\left(\mathrm{p}=9.70 \mathrm{e}^{-06}\right)$ had higher species richness than uninfected (Figure 2C), including those infected at

209 light [eggs per gram (epg) < 999; $\mathrm{p}=0.045$ ] and moderate $\left(\mathrm{epg}<9,999 ; \mathrm{p}=8.34 \mathrm{e}^{-07}\right)$ intensities

210 (Figure 2C). Other alpha diversity indices (i.e., Shannon and Simpson) are shown in Figures S10A-

$211 \mathrm{H}$ and results for each village are shown in Figures S11A-E. The prevalence of helminth infection

212 varied according to village and was highest in Rasau (89.6\%, $n=43$ of 48), followed by Legong

$213(81.0 \%, 81$ of 100), Judah (71.6\%, 83 of 116), Sepat (55.0\%, 22 of 40) and Bangkong (14.9\%, 7

214 of 47) (Figure 2D). As Trichuris was the predominant helminth, the prevalence of Trichuris was

215 similar for Rasau (81.3\%, 39 of 48), Legong (77.0\%, 77 of 100), Judah (65.6\%, 76 of 116), Sepat

$216(50.0 \%, 20$ of 40) and Bangkong (10.6\%, 5 of 47) (Figure 2D). There was no one infected with

217 helminths in KL. The two villages with the highest prevalence, Rasau $(p=0.0002)$ and Legong ( $p=$

218 8.1 $\mathrm{e}^{-07}$ ), showed higher species richness compared to KL (Figure 2E). Also, we observed that

219 species richness appeared to be greater when helminth infections in the villages were more

220 prevalent, which was similar to the order of villages for unmapped reads shown in Figure 1D. To

221 determine if Trichuris infection intensity was associated with unmapped reads, we performed a

222 Spearman correlation test and found that the intensity of Trichuris infection was positively

223 correlated $(\mathrm{p}=3.2 \mathrm{e}-06, \mathrm{R}=0.25)$ with the percentage of unmapped reads to the HRGM database

224 (Figure 2F). These results indicated that helminth infections were associated with under-

225 representation in the catalog of bacterial genomes.

226 We next determined the relative contribution of village and helminth infection status on

227 the gut microbiome in relation to other factors (e.g., if they had probiotic food, diarrhea, and 
antibiotics drug in the past 3 months, different age groups, subtribes and protozoa infection). ADONIS analysis indicated that only village $\left(p=1.000 \mathrm{e}^{-4}, \mathrm{~F}\right.$ value $\left.=1.672, \mathrm{R}^{2}=0.025\right)$ and helminth status $\left(\mathrm{p}=0.028, \mathrm{~F}\right.$ value $\left.=1.387, \mathrm{R}^{2}=0.010\right)$ had significant effects on the gut microbiome 231 composition (Figure 2G). Since village has the largest effect size on gut microbiome composition, 232 we next used MaAsLin2 [28] to identify bacterial taxa that were differentially abundant between

233 Trichuris infected and uninfected individuals from specific villages. Importantly, we found that 234 the bacterial species that were most differentially abundant between infected and uninfected 235 subjects were unique to specific village (Figure 2H). For example, 236 Haemophilus_A.parahaemolyticus and Corynebacterium.provencense were different in Bangkong 237 and Rasau, whereas Prevotella. sp900316565 was different in Sepat; C941.sp004557565 and 238 UBA10281.HRGM_Genome_2392 in Judah; Prevotella.sp900546575 and 239 Prevotella.HRGM_Genome_3676 in Legong; UBA1829.sp900549045 and 240 F082.HRGM_Genome_5331 in Rasau (Figure 2H). Similar patterns of results were obtained with 241 ANCOM-BC (Figure S12). These results indicated that helminth infections may have different 242 effects on the gut microbiome in different villages.

\section{Dynamic changes to the gut microbiome after anthelminthic treatment}

245 Longitudinal interventional approaches provide stronger assessment of cause-and-effect 246 relationships. Fecal samples analyzed at Pre- and Post-anthelminthic treatment provided insights 247 into the effects of deworming on the gut microbiome. Individual subjects were grouped into 4 248 categories [i.e., Full responders (n=43 paired; from 26-33,099 epg to 0 epg), Partial responders 249 ( $\mathrm{n}=23$ paired; from 281- 119,875 epg to 26-71,579 epg), Non-responders ( $\mathrm{n}=5$ paired; from 204250 1,097 epg to 281-1,632 epg) and Uninfected ( $\mathrm{n}=58$ paired)], based on the Trichuris infection 
A

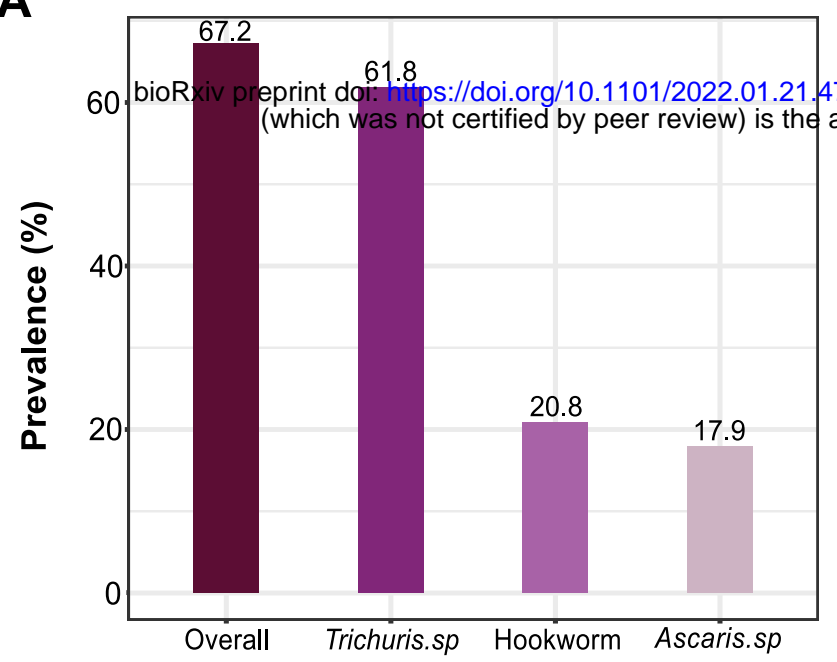

C
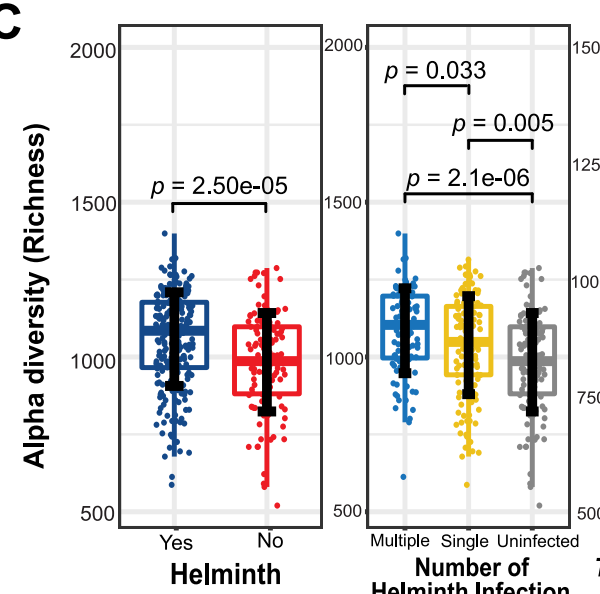

Helminth Infection
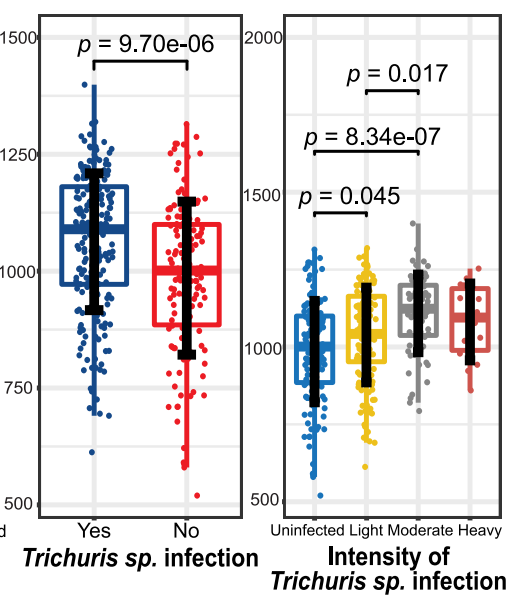

$\mathbf{E}$

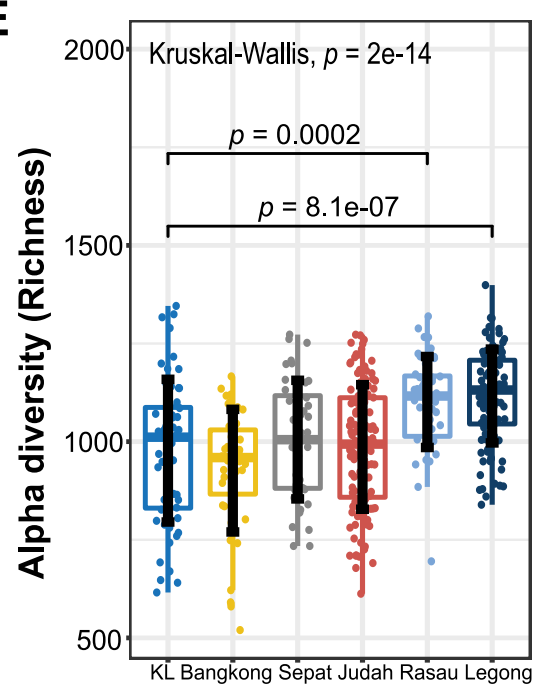

$F$

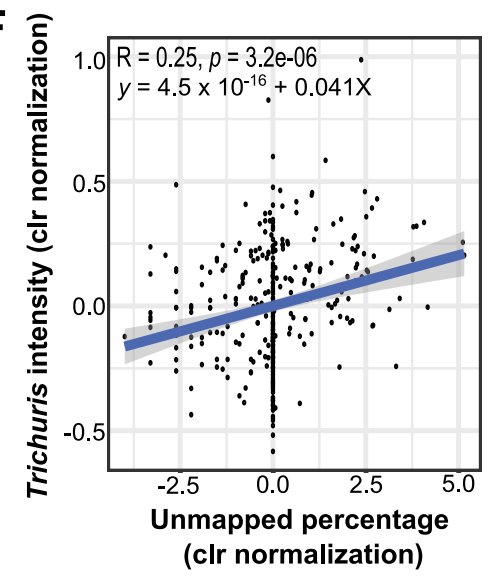

G

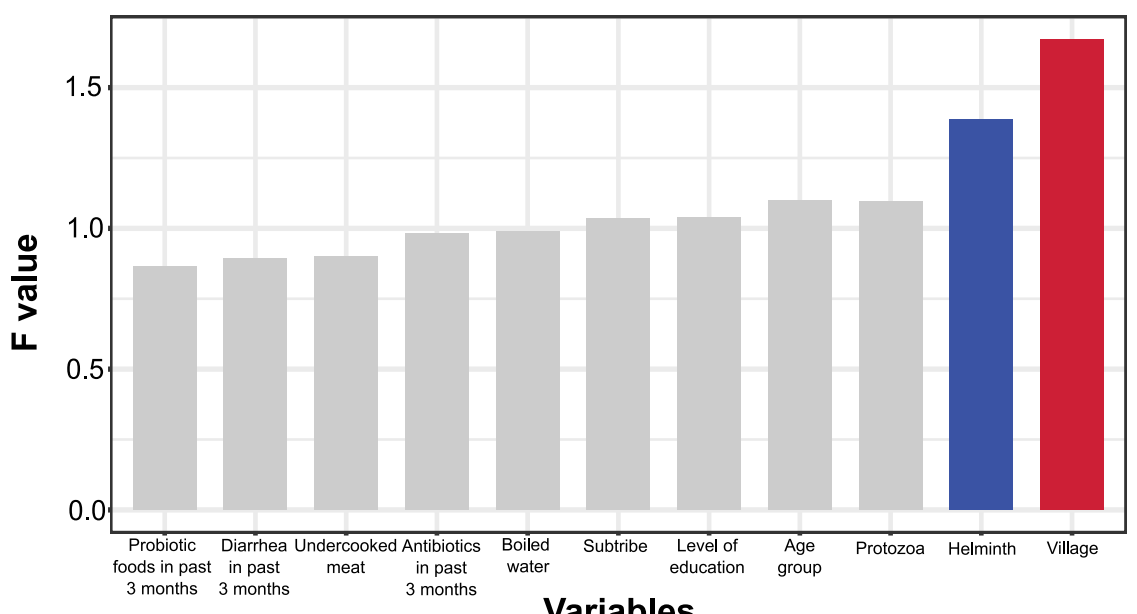

$1 \longdiv { 0 0 0 8 8 0 0 0 }$

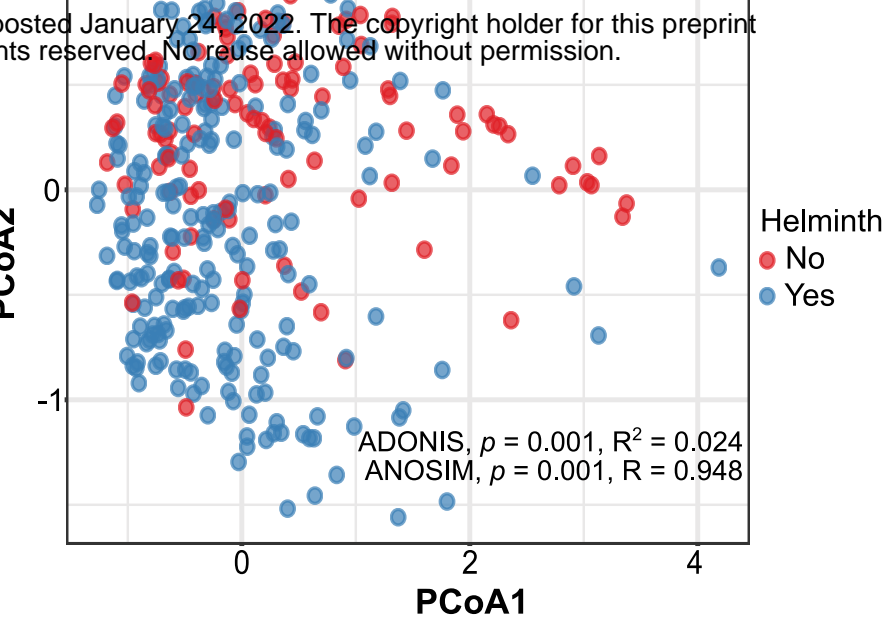

D
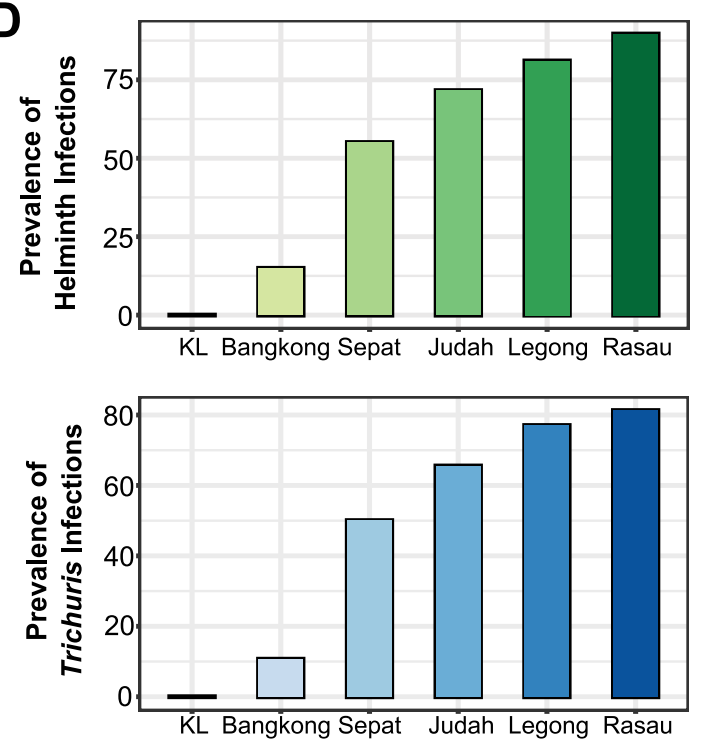

H

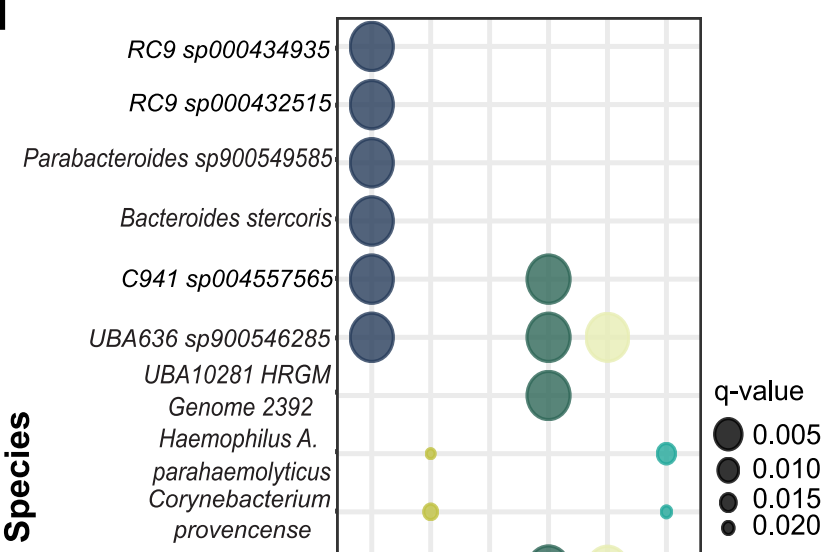

UMGS1585 sp900553205

UBA10281 HRGM Genome 2385 Prevotella sp900316565 CAG488 sp000434055 Prevotella sp900546575

Prevotella HRGM

Genome 3676 UBA1829 sp900549045 F082 HRGM Genome 5331 All Bangkong Sepat Judah Legong Rasau
Villages 
Fig. 2 Effects of intestinal helminth infection status on gut microbial diversity and composition for the 407 Orang Asli individuals.

253 A The prevalence of intestinal helminth infection in the OA cohort based on overall infection

254 status, as well as specific intestinal helminth infection (i.e., Trichuriasis, ascariasis and hookworm

255 infection). B Principal Coordinates Analysis (PCoA) of Jaccard distances based on gut microbiota

256 profiles of the OA cohort. The individuals infected and uninfected with intestinal helminths are

257 denoted by blue and red respectively (ADONIS: $\mathrm{p}=0.001, \mathrm{R}^{2}=0.024$; ANOSIM: $\mathrm{p}=0.001$,

$258 \mathrm{R}=0.948)$. C Alpha diversity boxplot of species richness based on different status of intestinal

259 helminth infection, number of intestinal helminth infection, Trichuris infection and intensity of

260 Trichuris infection. Wilcoxon rank sum test is used for two independent variables while the

261 Kruskal-Wallis test is used for more than two comparison groups. D The prevalence of intestinal

262 helminth infection (Top) and Trichuris infection (Bottom) by different geographical locations. E

263 Comparison of alpha diversity (species richness) between individuals from KL and specific OA

264 villages. F Spearman correlation between the intensity of Trichuris infection and percentage of

265 unmapped reads to the HRGM database $\left(\mathrm{p}=3.2 \mathrm{e}^{-6}, \mathrm{R}=0.25\right)$. The blue line represents the linear

266 regression between intensity of Trichuris infection and percentage of unmapped reads. G Bar plot

267 of the F statistic values from ADONIS analysis of variables that contribute to the gut microbiota

268 composition. Colored bars indicate the variables that show significant effects on gut microbiota

269 variation $(\mathrm{p}<0.05)$. H Bubble plot of bacterial species that are differentially abundant between

270 Trichuris infected and uninfected individuals in all samples, as well as in specific village. The size

271 of the bubble is negatively proportional to the p-value.

272

273 
274 intensity before and after deworming (Figure 3A). While mixed infection was present in some

275 individuals, hookworm and Ascaris infection were always cured after deworming (Supplementary

276 Figure S13A).

277

First, we compared Pre and Post samples for Responders, which include both Full and

278 Partial responders. PCoA based on Jaccard distances showed that there are differences in gut

279 microbiota composition Pre and Post treatment, but the effect size was small (ADONIS: $\mathrm{p}=0.001$,

$280 \mathrm{R}^{2}=0.014$; ANOSIM: $\mathrm{p}=0.001, \mathrm{R}=0.072$ ) (Figure 3B, Supplementary Table S2). Since albendazole

281 may have a direct effect on the microbiota, we next compared the gut microbiota profile Pre and

282 Post treatment for helminth negative individuals. Similar to the Responders, PCoA based on

283 Jaccard distances also indicated differences in gut microbiota composition between Pre and Post

284 samples, with a small effect size (ADONIS: $\mathrm{p}=0.006, \mathrm{R}^{2}=0.012$; ANOSIM: $\mathrm{p}=0.001, \mathrm{R}=0.069$ )

285 (Figure 3C, Supplementary Table S2). NMDS ordination, Bray-curtis distance matrix and beta-

286 dispersion analysis showed similar results (Figures S14A-E and S15A-E, Table S2) and there were

287 no significant changes to alpha diversity at Pre and Post treatment (i.e., Richness, Shannon,

288 Simpson) (Supplementary Figures S13B and C).

289 Using MaAslin2 for differential abundance testing, we found changes of 911 bacterial

290 species at Pre and Post treatment among Responders. However, there was substantial overlap with

291 changes found in Pre and Post treatment samples for helminth negative individuals (658 species,

$29272.2 \%$ ) (Figure 3D and Supplementary Figure S16), with only 253 taxa which were specific to the

293 Responders. For example, in both Responders and uninfected individuals, the relative abundance

294 of Collinsella sp003466125 $\left(\mathrm{p}=1.52 \mathrm{e}^{-08} ; \mathrm{p}=3.66 \mathrm{e}^{-07}\right.$, respectively) and RUG013.sp001486445

$295\left(\mathrm{p}=2.53 \mathrm{e}^{-07} ; \mathrm{p}=3.80 \mathrm{e}^{-06}\right)$ was reduced after deworming while the relative abundance of Bilophila

296 sp900550745 increased $\left(\mathrm{p}=1.33 \mathrm{e}^{-08} ; \mathrm{p}=5.81 \mathrm{e}^{-05}\right)$ (Figures 3E and $\left.\mathrm{F}\right)$. Hence, albendazole may 
A

\section{B Responders}

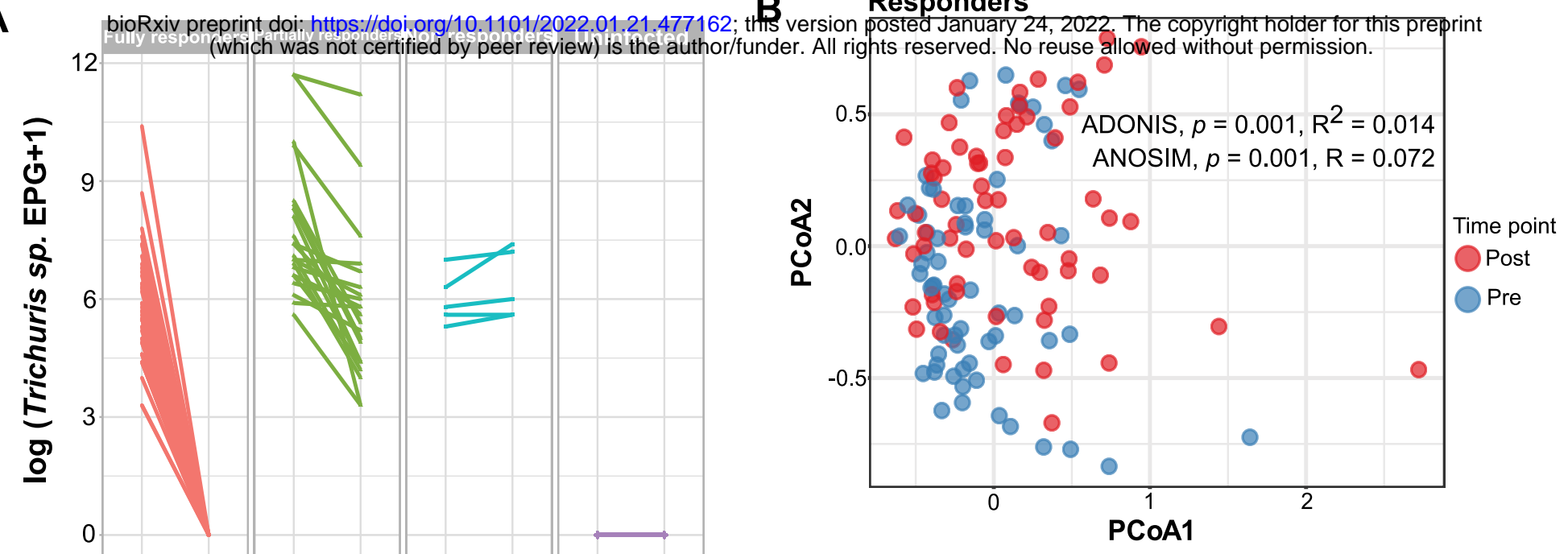

C

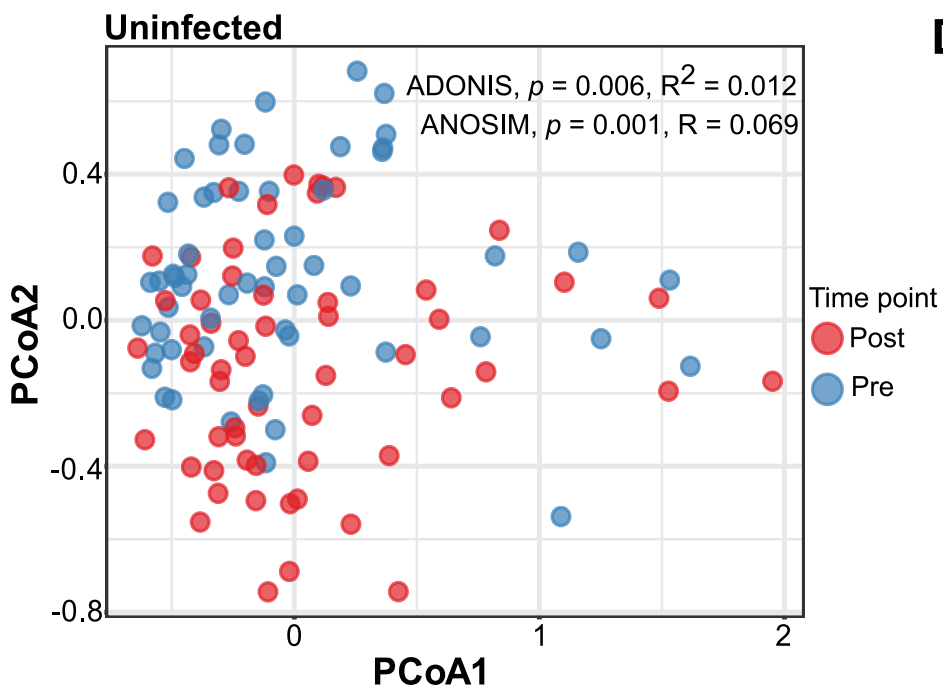

D Responders

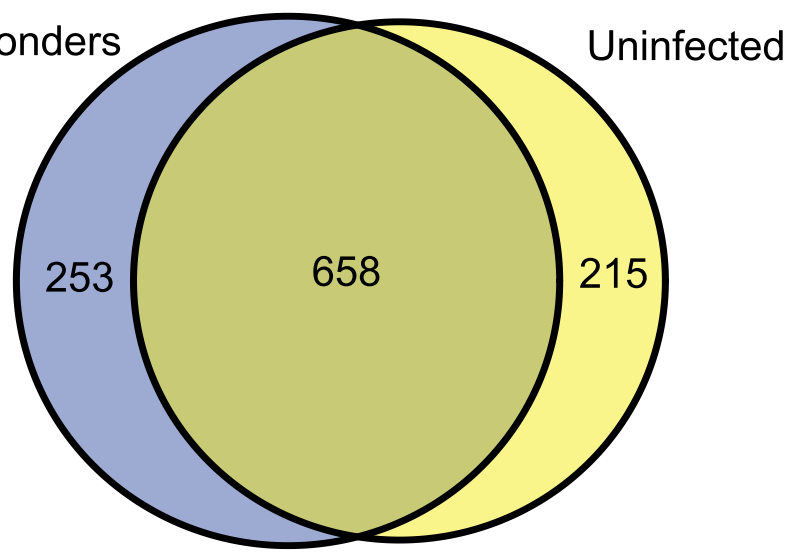

E
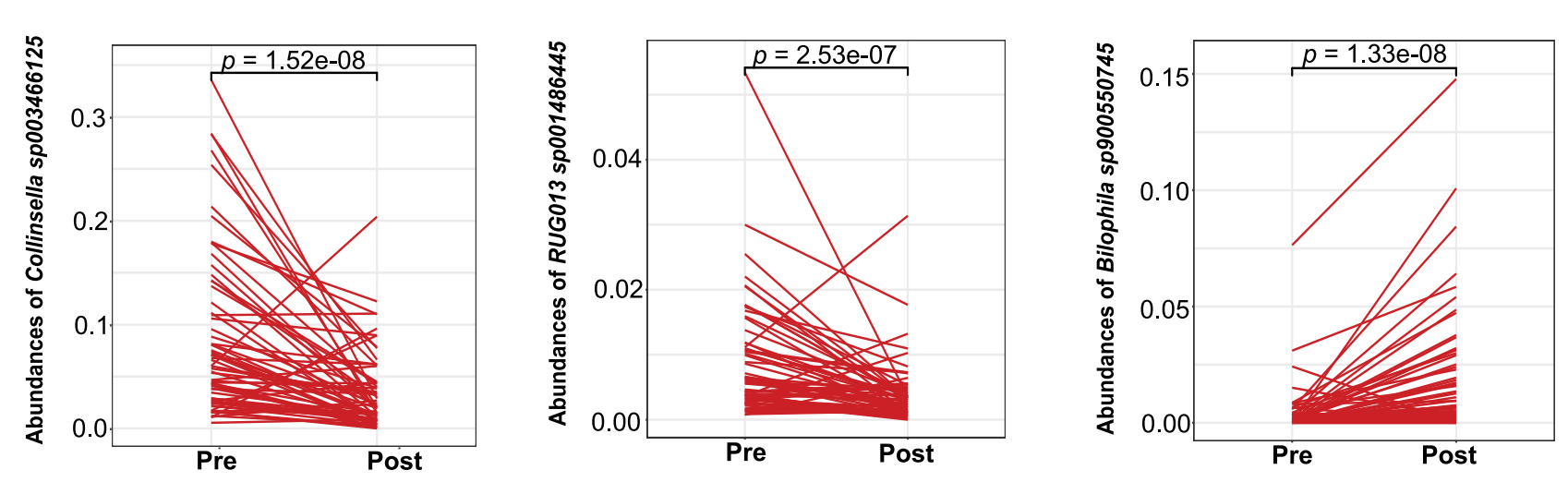

$\mathbf{F}$
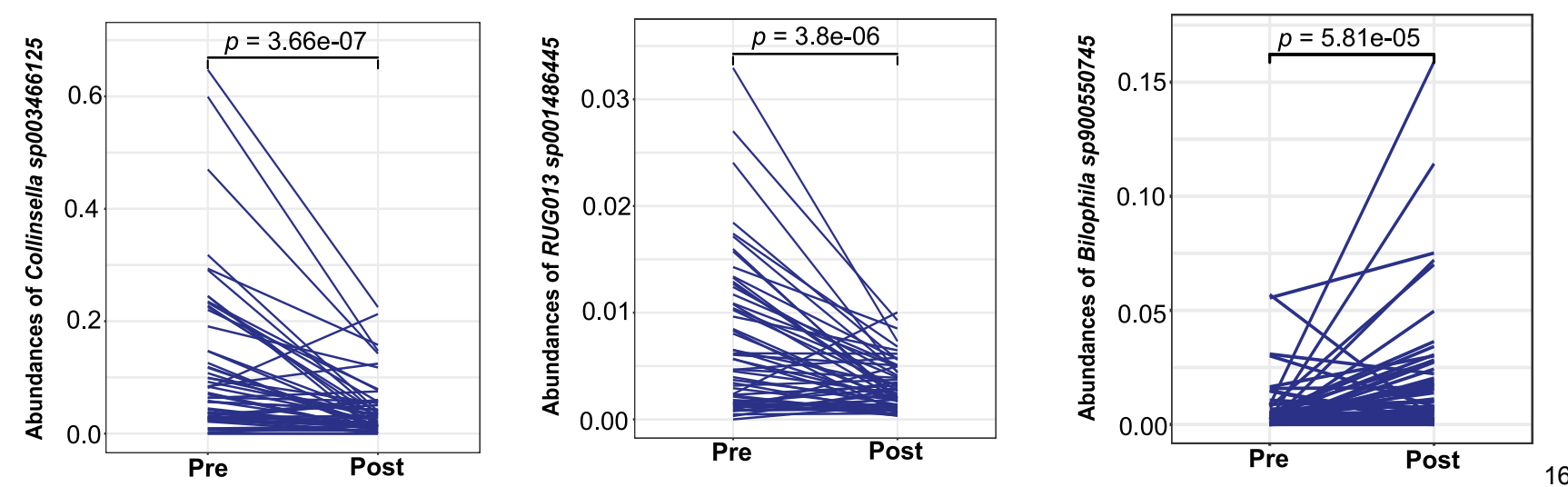

treatment.

A Line plots show changes of the infection intensity of Trichuris pre and post response to anthelminthic drugs stratified by Full responders $(n=43)$, Partial responders $(n=23)$, Nonresponders $(n=5)$ and Uninfected individuals $(n=58)$. B Principal Coordinates Analysis (PCoA) plot of Jaccard distances based on gut microbiota profiles of Responders (ADONIS: $\mathrm{p}=0.001$, $\mathrm{R}^{2}=0.014$; ANOSIM: $\mathrm{p}=0.001, \mathrm{R}=0.072$ ), with Pre-anthelminthic treatment (blue) and Postanthelminthic treatment (red). C Principal Coordinates Analysis (PCoA) plot of Jaccard distances based on gut microbiota profiles of Uninfected subjects (ADONIS: $\mathrm{p}=0.006, \mathrm{R}^{2}=0.012$; ANOSIM: $\mathrm{p}=0.001, \mathrm{R}=0.069)$ (Figure 3C, Supplementary Table S2), with Pre-anthelminthic treatment (blue) and Post-albendazole treatment (red). D Venn diagram depicting the number of shared and exclusive bacteria species that are found to be differentially abundant (Pre and Post) between Responders and Uninfected individuals. The blue area includes 253 bacteria that are altered only in Responders while the yellow and mixed color area indicates the 873 bacteria that are altered in

311 Uninfected individuals. E \& F Line plots showing changes to 3 of the the top differentially

312 abundant bacterial species between Pre and Post treatment samples from $\mathbf{E}$ Responders and $\mathbf{F}$

313 Uninfected individuals, with p-values determined by the Wilcoxon signed-rank test. Pre Pre-

314 anthelminthic treatment, Post Post-anthelminthic treatment. 
320 have a substantial effect on the microbiota that may be an important confounding factor for

321 deworming studies.

322 In some individuals, we conducted a follow-up study 42-Days post-anthelminthic

323 treatment. There were no differences in alpha diversity on Day-42 (Figure S17A) and although

324 beta diversity analysis showed significant differences between three time points (i.e., Pre, 21-Day, 325 and 42-Day) (Supplementary Figures S17B-C, Supplementary Table S3), these differences are 326 driven by the pre-treatment samples (Supplementary Figure S17D). Therefore, the changes in the 327 gut microbiome in both Responders and uninfected individuals after albendazole treatment remain 328 stable by Day-42.

\section{Bacterial replication in the context of helminth infection}

331 Actively replicating bacteria can be inferred based on calculating an index of replication from 332 metagenomic sequencing for coverage trends resulting from bi-directional genome replication 333 from a single origin of replication. We used the algorithm Growth Rate Index (GRiD) to estimate 334 the growth rate of gut bacteria in relation to helminth infection status. We found that the growth 335 rate of 61 bacterial species was associated with intestinal helminth infection (Figure 4A; 336 Supplementary Table S4). Next, we conducted Spearman correlation analysis on Trichuris egg

337 burden with the growth rate of these 61 bacterial species to identify the most highly correlated 338 bacteria. From the 61 bacterial species, there were 11 bacterial species correlated to Trichuris egg 339 burden (Figure 4B and Supplementary Figure S18A), with Catenibacterium sp. being positively 340 correlated $\left(\mathrm{p}=2.83 \mathrm{e}^{-04}, \mathrm{R}=0.44\right)$ (Figure 4C) while Bacteroides vulgatus was negatively correlated $341(\mathrm{p}=0.003, \mathrm{R}=-0.39)$ (Supplementary Figure S18B). The growth rate of Catenibacterium sp. was 
342 notably higher in Trichuris infected individuals $\left(\mathrm{p}=1.65 \mathrm{e}^{-16}\right)$. Conversely, the growth rate of

343 Bacteroides vulgatus was lower in Trichuris infected individuals ( $\left.\mathrm{p}=2.65 \mathrm{e}^{-16}\right)$ (Figure 4D).

344 For the longitudinal deworming component of the study, we observed that the growth rate

345 of 12 bacterial species were different between Pre and Post treatment samples among the

346 Responders. Among these bacterial species, half of them (i.e. Bilophila wadsworthia,

347 Lachnospiraceae bacterium_7_1_58FAA, Bacteroides vulgatus, uncultured Oscillibacter sp.,

348 Clostridium sp.CAG.81, Oscillibacter sp.57_20) were also identified from the cross-sectional

349 analysis (Supplementary Figure S18C). Spearman correlation analysis on Trichuris burden with

350 the growth rate of these bacterial species demonstrated that uncultured Oscillibacter sp. $(\mathrm{p}=0.037$,

$351 \mathrm{R}=-0.29)$ and Bacteroides vulgatus $(\mathrm{p}=0.038, \mathrm{R}=-0.24)$ were negatively correlated with Trichuris

352 burden (Supplementary Figure S18D), indicating more replication in Responders after

353 anthelminthic treatment (Supplementary Figure S18E). However, some of these relationships

354 (Firmicutes bacterium CAG.102, Lactobacillus rogosae, Clostridium sp. CAG.81 and Bacteroides

355 vulgatus), were also observed in the helminth negative group (Supplementary Figure S18F).

356 Hence, it could be difficult to disentangle the effects of helminth infection and direct effects of

357 albendazole treatment on the dynamics of the microbiome.

\section{Discussion}

360 In this study, we examined 650 stool metagenomes from a cohort of 351 indigenous Malaysians

361 from 5 villages with different prevalence (14.9\%-89.6\%) of helminth infections, along with 56

362 urban citizens (uninfected) living in Kuala Lumpur city. To our knowledge, this is the largest study

363 utilizing shotgun metagenomics to investigate the interactions between helminth infection and the

364 human microbiome. 


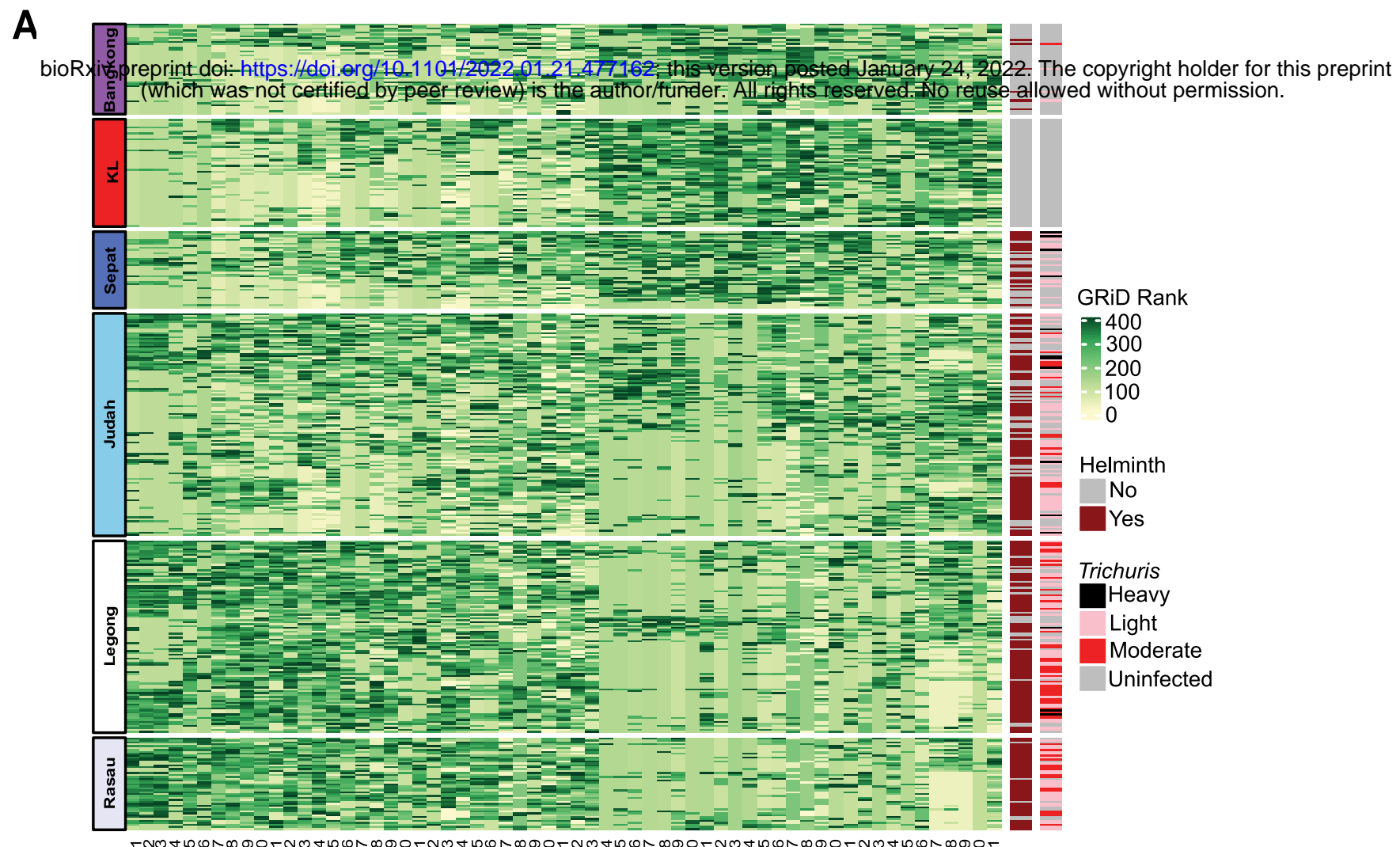

B

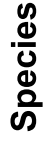

Lachnospiraceae bacterium 7_1_58FAA

Bifidobacterium longum

Bacteroides vulgatus

$-0.25$

Spearman's rank correlation coefficient
Correlation with Trichuris intensity

Negative

Positive
D

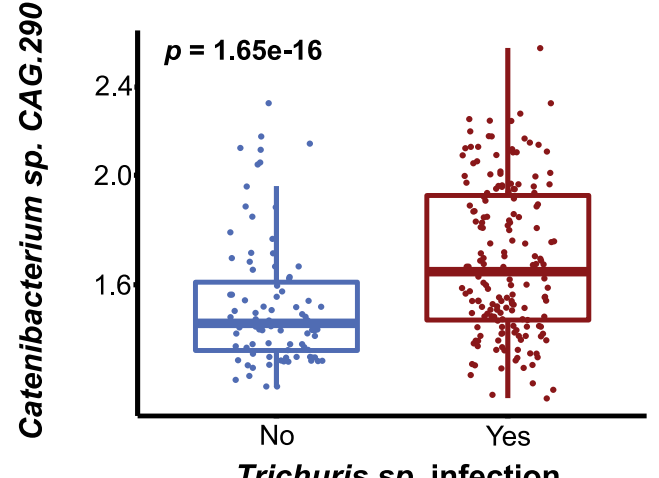

Trichuris sp. infection

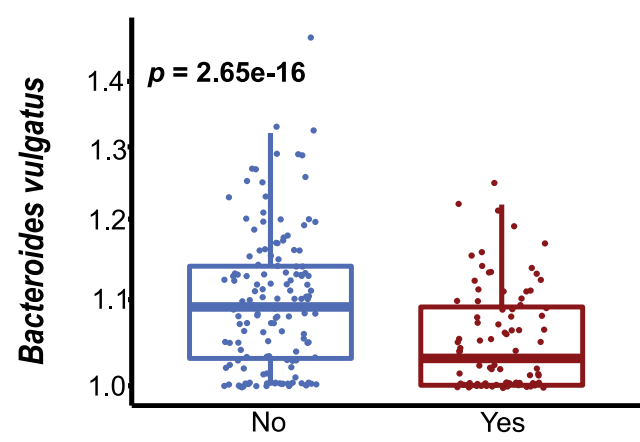

Trichuris sp. infection 
Fig. 4 Gut bacterial replication in the context of intestinal helminth infection.

366 A Heatmap of the Growth Rate Index (GRiD) score, which infers an index of replication for gut

367 bacteria in relation to helminth infection status of individuals. Samples are shown in rows, by

368 village, whereas the GRiD score of each bacterium are shown in columns. The first vertical side

369 bar color codes the intestinal helminth infection status while the second side bar indicates the

370 infection intensity of Trichuris. B GRiD score correlation between bacterial species with the

371 infection intensity of Trichuris. The bar chart shows the Spearman's rank correlation coefficient.

372 Blue and grey colors represent the positive and negative correlations respectively. C Spearman's

373 rank correlation between Catenibacterium sp. growth rate and the infection intensity of Trichuris

$374\left(\mathrm{p}=2.83 \mathrm{e}^{-4}, \mathrm{R}=0.44\right)$. D Boxplots of GRiD score for Catenibacterium sp. and Bacteroides vulgatus

375 in intestinal helminth infected and uninfected individuals. P-values are calculated by Wilcoxon

376 rank sum test for cross-sectional comparison, Wilcoxon signed-rank test for longitudinal samples,

377 with Benjamini-Hochberg correction. 
We found that mapping onto HRGM, which incorporates MAGs, improved taxonomic classification substantially, especially for the indigenous Orang Asli. We also found that the microbiota is dominated by Firmicutes A, which is represented by mostly uncultured bacteria, highlighting the under-representation of cultured bacteria from indigenous groups. This could be

391 an important caveat for most of the previous studies on helminths and the gut microbiota, which

392 were conducted using 16s rRNA sequencing [11-13, 17, 29] with the taxonomic classification 393 based on mapping to the reference databases Greengenes, SILVA and Ribosomal Database Project 394 (RDP). In a recent shotgun metagenomic study on 175 Cameroonian samples, the data was also 395 mapped onto a reference database from NCBI [30] and the investigators noted that the 396 classification of the relative abundance of bacteria did not correspond to data from 16S Greengenes 397 classifications for the V4 region [30]. A different study also indicated that 16s rRNA gene 398 sequencing only provided a portion of the gut microbiota profile compared to shotgun 399 metagenomics [31]. Hence, we suggest assembling the Malaysian reference catalog to provide 400 substantial benefit for future microbiome studies, especially from under-represented geographic 401 regions and for rural and indigenous populations.

402 In this metagenomic study, we found that intestinal helminth infection status was 403 associated with higher species richness, which was consistent with our previous findings and 404 others conducted using 16s rRNA sequencing [10, 12, 13, 17, 30, 32]. However, we did not find a 405 significant difference at Pre and Post deworming, which could be because of smaller sample size 406 and could also be confounded by the effects of albendazole. Additionally, other studies have not 407 observed an effect of helminths on microbial diversity $[8,11,14,17,18,33]$. It is important to 408 note that each study cohort has different prevalence rates for different helminth species, as well as 409 distinct genetics and living conditions. This study has a larger sample size than our previous studies 
$410[13,34]$ and enabled us to examine the interactions of helminth infection and the gut microbiome

411 in different villages. Indeed, village has the largest effect size on gut microbiome variation,

412 followed by helminth infection status. Notably, villages with higher helminth prevalence rates also

413 have higher microbial diversity, but in different villages, helminth infection is associated with

414 differential abundances of distinct bacterial taxa. It is important to note that the different villages

415 represent diverse environments, practicing unique lifestyles and have different hygiene practices.

416 Compared to other villages, Rasau and Legong villages (with higher helminth prevalence) are

417 located near the forest with high exposure to the soil-environment, which may harbor more

418 microbes [35] and mouse experiments showed that exposure to soil increases gut microbiota

419 diversity [36]. From our questionnaire, a higher percentage of villagers from Rasau and Legong

420 are plantation agricultural workers (Rasau: 30.4\%; Legong: 14.8\%; Others: < 6.7\%), do not have

421 a toilet $(52.5 \% ; 20.8 \% ;<13.0 \%)$, and practice open defecation $(46.5 \% ; 32.0 \% ;<7.4 \%)$ more than

422 other villages. As Trichuris eggs germinate in the soil this may increase exposure to Trichuris, as

423 well as other microbes in the contaminated soil, resulting in higher microbial diversity in different

424 settings.

425 We also found that deworming helminth negative individuals can influence the gut 426 microbiome that overlaps substantially with changes in individuals responding to drug treatment 427 by having reduced worm burdens. This indicates that albendazole may directly affect the 428 microbiome, or that there are population effects that can influence uninfected people. There are 429 four previous studies on albendazole $[10,11,14,17]$. The first study conducted among Ecuador 430 school children did not find any difference in bacterial composition among both Trichuris infected 431 and uninfected groups after a combination of albendazole and ivermectin treatment [14]. In 432 contrast, the second study in Indonesia found an increase of Actinobacteria and Bacteroidetes with 
433 albendazole treatment versus placebo in individuals that remained helminth-infected post-

434 treatment, but not in uninfected individuals [17]. In addition, Rosa and coworkers also

435 demonstrated that the gut bacterial composition was altered in a helminth-uninfected group in

436 Indonesia after two years of albendazole treatment [10]. Another study in Kenya found

437 significantly reduced Chao richness for uninfected individuals after deworming treatment,

438 suggesting an effect of albendazole [11]. Albendazole is a prodrug that metabolizes rapidly to

439 albendazole sulfoxide (the active anthelminthic compound) and albendazole sulphone (the inactive

440 compound). Some bacterial species (Enterobacter aerogenes NCIM 2695, Klebsiella aerogenes

441 NCIM 2258, Pseudomonas aeruginosa NCIM 2074 and Streptomyces griseus NCIM 2622) could

442 be involved in metabolizing albendazole to albendazole sulfoxide and albendazole sulphone [37].

443 Albendazole can also be metabolized by the resident microbiota in gut rumens in sheep and cattle

444 [38]. Hence, the gut microbiota could play a crucial role in metabolizing albendazole and influence

445 drug bioavailability and efficacy on infected individuals. Why albendazole has lower efficacy

446 against Trichuris infection than hookworm and Ascaris needs further investigation [39]. Future

447 studies could apply metabolomics profiling to investigate metabolite differences between response

448 groups to better understand the underlying mechanisms.

450 Methods

451 Study design and sample collection

452 This study consists of both cross-sectional and longitudinal phases. Cross-sectional comparisons 453 were made on the Orang Asli cohort (OA) and between OA and urban cohorts (KL) living in the 454 capital city of Malaysia, Kuala Lumpur. Within the Orang Asli community, we studied five Orang 455 Asli villages: 1. Rasau village (Perak state); 2. Judah village (Selangor state); 3. Sepat village 
(Selangor state); 4. Bangkong village (Selangor state) and 5) Legong village (Kedah state). The locations of each village are displayed on a map using ArcGIS (version 10.7.1) together with other information including states, tribes and subtribes (Supplementary Figure S1). A total number of 351 samples were collected from Orang Asli subjects and 56 samples from KL subjects in this cross-sectional component (aged 4 years old and above) (Supplementary Figure S2). 400mg albendazole for 3 consecutive days after the first stool sample collection. Stool samples were collected from the treated subjects at 21-days and 42-days following anthelminthic treatment.

464 However, due to the restriction during the COVID-19 pandemic, only 4 Orang Asli villages were 465 included in this phase, excluding Legong village. There was no follow-up for urban controls after 466 the cross-sectional phase because they were not treated with albendazole. Sample selection for 467 analysis was based on a complete set of paired stool samples [Pre (Pre-anthelminthic treatment) 468 and Post (21-day post-anthelminthic treatment)] $(n=129)$ and 3 time-points stool samples 469 collection (Pre, 21-day and 42-day) $(n=110)$. Four subject samples were removed from the 470 longitudinal analysis due to incomplete data collection. Then, subjects were categorized into three 471 groups for comparison: Responders, Non-responders and Uninfected, based on their infection 472 status before and after the albendazole treatment. Responders ( $n=66$ paired samples) refer to 473 individuals who were positive at baseline and became negative or showed reduction of infection 474 intensity after deworming. Non-responders ( $\mathrm{n}=5$ paired samples) refer to individuals who were 475 positive at baseline and showed increment or maintain of egg counts after deworming. Uninfected 476 ( $\mathrm{n}=8$ paired samples) refer to negative individuals before and after the treatment. Non-responders 477 were not be included in the gut metagenome analysis due to insufficient sample size. The details 478 number of samples collected at each time points were shown in Supplementary Figure S2. 


\section{Fecal sample preparation and analysis}

480 All the stool samples collected were divided into two portions: (i) preserved in $2.5 \%$ potassium 481 dichromate and stored at $4^{\circ} \mathrm{C}$ for intestinal helminth infection screening and (ii) aliquoted in $1.5 \mathrm{ml}$

482 cryovial tube, frozen immediately in dry ice and kept at $-80^{\circ} \mathrm{C}$ for shotgun metagenomic analysis 483 (Supplementary Figure S19). To detect and quantify helminth infections, Kato-Katz was

484 performed. A thick smear was prepared from the fresh stool according to the manufacturer's 485 instructions (Kato-Katz kit, Mahidol University, Thailand) [40]. Infection intensity was stratified 486 into light, moderate or heavy according to WHO cut-offs [41]. Formalin ether sedimentation was 487 performed according to [42]. Stool samples were considered positive if any soil-transmitted 488 helminths were detected from any of these two methods. DNA was extracted from stool samples 489 using Qiagen DNeasy PowerSoil Pro kit (Qiagen, Hilden, Germany). DNA library were prepared 490 using Illumina TruSeq DNA Nano Library kit (Illumina, United States). Paired end metagenomic 491 sequencing was performed on the NovaSeq6000 S4 platform to generate an average of 20 million 492 paired end reads per sample (range 13-35 million paired end reads), with a read length of 150bp 493 and insert size of 350bp.

\section{Sequencing analysis pipeline}

495 The overall bioinformatic analysis workflow from pre-processing to downstream analysis is shown 496 in Supplementary Figure S3. In brief, the whole process of quality filtering and trimming of the 497 raw sequence reads was performed by using KneadData (version 0.7.4) integrated with 498 Trimmomatic [42], Bowtie [43] and FastQC [44] tools. Sequence reads were trimmed by using 499 Trimmomatic with default settings, based on a sliding window trimming approach 500 (SLIDINGWINDOW:4:20) when average base Phred quality score over four reads dropped below 50133 (PHRED 33). Next, sequence reads were mapped against human reference genome (hg37) using 
502 Bowtie2 with default parameters (very-sensitive end-to-end alignment) to remove human host

503 genome. The filtered reads were then used for the following analysis. Additionally, FastQC was

504 used to perform quality checks on the raw metagenomic reads before pre-processing and after pre-

505 processing to ensure of high-quality metagenomic reads for downstream analysis.

For taxonomic classification, Kraken2 (version 2.1.0) [45], a k-mer matching algorithm

507 classifier was used for assigning taxonomic labels to the trimmed reads. The trimmed reads were

508 mapped using Kraken2 against a standard database (1) RefSeq database (bacterial, protozoa, fungi,

509 viral and archaeal) and two MAGs integrated databases (2) Human Reference Gut Microbiome

510 (HRGM) database, with 232,098 reference genomes [27], (3) The Unified Human Gastrointestinal

511 Genome (UHGG) database, with 204,938 reference genomes [24]. After taxonomic classification

512 by Kraken2, Bayesian Re-estimation of Abundance with KrakEN (Bracken2) (version 2.6.0) [46]

513 was used to compute the relative abundance of bacterial for each taxa (from phylum to species

514 level) using a read length parameter of 150. The mapped reads of the OA and KL cohorts were

515 then plotted into a violin plot using ggplot2 package [47] to access which databases provide better

516 taxonomic classification. The distribution of the mapped reads was determined using the Shapiro

517 test from the rstatix package [48]. Then, the difference between the mapped reads of KL was

518 determined using the Wilcoxon rank sum test from ggplot2 package [47]. The data generated from

519 Bracken2 were exported in the form of BOIM (Biological Observation Matrix) table and analyzed

520 using R programming language (version 4.0.5, R Studio, Inc., Boston, MA, USA). The BIOM

521 table was imported and filtered using the phyloseq package [49]. Only those taxa with a minimum

522 abundance of $20 \%$ across all the samples and a minimum coefficient of variation of 3.0 were

523 included in the following analysis (Supplementary Figure S20). In general, ggplot2 [47] and

524 ggpubr package [50] was used to create visualization plots. 
526 kmers, the low abundance of k-mers were trimmed using "trim-low-abun" from khmer project,

527 with a k-mer abundance cut-off of 3.0 and trimming coverage of 18. Signatures were generated

528 for each sample using "sourmash compute" with a compression ratio of 10,000 (-scaled 10,000)

529 and $\mathrm{k}-\mathrm{mer}$ lengths of 21, 31 and $51(-\mathrm{k} 21,-\mathrm{k} 31,-\mathrm{k} 51)$. A signature output was generated for Jaccard

530 distance comparisons. Before the k-mer comparison, "sourmash index" was used to create a

531 Sequence Bloom Tree (SBT) database from a collection of signatures. Lastly, "sourmash compare"

532 was used with default settings to compare the signatures at each length of $\mathrm{k}$.

533 The core microbiota was determined by including taxa present across all samples (i.e,

534 abundance of $100 \%$ across all the samples). Random Forest (randomForest package) was used to

535 identify microbiome taxa predictive of OA and KL [52] groups. Because of imbalance in the

536 number of OA ( $\mathrm{n}=594)$ and KL (n=56) samples, a "SMOTEd" (consist of 280 OA and 336 KL)

537 data set was generated using the package DMwR [53] to counter this issue [54]. After that, the

538 Random Forest model was built based on this "SMOTEd" data set, and tuned with the methods

539 described by Brwonlee (2016) [55], followed by the significant testing using the methods

540 described by [56].

541 Alpha diversity, in terms of species richness [57], Shannon [58] and Simpson index [59],

542 were analyzed using the microbiomeSeq package [60]. The Wilcoxon rank sum test [61] was

543 performed to compare groups statistically in the cross-sectional study (i.e., Helminth infected vs

544 Uninfected and OA villages versus the KL) whereas Wilcoxon signed-rank test was used for paired

545 samples in longitudinal study (i.e. Pre vs Post for both Responders and Uninfected). The

546 Spearman's rank correlation test [62] between the intensity of the Trichuris infection and the 
547 microbiome's species richness was conducted after the data were normalized via centred log ratio

548 transformation using the compositions package [63] .

549 Beta diversity analysis was performed on both the Jaccard and Bray-Curtis dissimilarity

550 matrix calculated from the taxon abundance data standardized using Hellinger. Differences in beta

551 diversity between groups (i.e., different OA villages and different helminth infection status) or

552 between different time points (Pre vs Post) were displayed with principal coordinates analysis

553 (PCoA) plots and non-multi-dimensional scaling (NMDS) plots. The comparison on pair-wise

554 distance of the samples between OA villages and KL was conducted using Wilcoxon rank sum

555 test. This same analysis was also applied to the output generated from K-mers sketches.

556 Permutational multivariate analysis of variance (PERMANOVA) under ADONIS function

557 [64] from the vegan package was conducted with 10,000 permutations on both the Jaccard and

558 Bray-Curtis dissimilarity matrix. This analysis was first performed on specific variables of interest

559 (i.e., different geographical locations, helminth status and Pre vs Post). ADONIS was used to

560 assess the effect of multiple variables on the gut microbial composition (e.g., if they had probiotic

561 food, diarrhea, and antibiotics drug in the past 3 months, different age groups, subtribes and

562 protozoa infections), as well as Analysis of similarity (ANOSIM) [65]. To test for multivariate

563 dispersions among groups, the permutation multivariate analysis of dispersion (PERMDISP) [64]

564 was performed via the betadisper function and Tukey test under the Vegan package [66].

Differential abundance analysis was performed using Multivariate Association with Linear

566 Models 2 (MaAsLin2) [28] for both cross-sectional (i.e., Trichuris infected vs non-infected for all

567 villages and specific village) and longitudinal data (i.e., Pre vs Post for Responders and

568 Uninfected). Analysis of composition Microbiomes with Bias Correction (ANCOM-BC) [67] was

569 also conducted to validate the output generated from MaAsLin2, for cross-sectional data only. The 
570 Wilcoxon rank sum test and Wilcoxon signed-rank test from the rstatix package [48] were used to

571 determine the p-value between groups for specific taxa.

572 Growth rate index (GRiD) (version 1.3) was used to evaluate the growth rate of microbial

573 species in metagenomic samples [68]. Samples were mapped to a GRiD database

574 (ftp://ftp.jax.org/ohlab/GRiD_environ_specific_database/stool_microbes.tar.gz), a stool-specific

575 database created based on microbes mostly found in stool. GRiD score > 1.02 indicates bacteria

576 are in growth phase whereas GRiD score $<1.02$ indicates that bacteria are in stationary or lag

577 phase. Bacterial species with GRiD score $<1.02$ in more than $75 \%$ of samples were filtered from

578 further analysis, and then filtered using a cut-off of 2.0 of coefficient of variation (Supplementary

579 Figure S21).

580 Cross-sectional comparison of bacterial species growth rate between helminth infected and

581 uninfected individuals was computed using Wilcoxon rank sum test and corrected using

582 Benjamini-Hochberg with false discovery rate (fdr) of 5\%. Bacterial taxa that significantly differed

$583(p<0.05)$ in GRiD score between helminth infected and uninfected individuals were displayed by

584 heatmap. The top 20 taxa with the lowest p values were identified for Spearman's rank correlation

585 test with Trichuris infection intensity. This analysis was also applied to the longitudinal study to

586 investigate the microbial growth rate between Pre and Post anthelminthic treatment among

587 Responders and Uninfected individuals.

\section{Key Resources Table}

589 List of the bioinformatic tools and R packages used are displayed in the table below:

\begin{tabular}{|l|l|l|l|}
\hline Tasks/analysis & Name & Source & Identifier \\
\hline
\end{tabular}




\begin{tabular}{|c|c|c|c|}
\hline $\begin{array}{l}\text { Bioinformatic } \\
\text { Tools }\end{array}$ & & & \\
\hline \multirow{4}{*}{$\begin{array}{l}\text { Quality control, } \\
\text { filtering and } \\
\text { trimming of raw } \\
\text { sequence }\end{array}$} & KneadData & No publication & $\begin{array}{l}\text { https://github.com/biobakery/kneadda } \\
\text { ta }\end{array}$ \\
\hline & Trimmomatic & $\begin{array}{l}\text { Bolger et al., } \\
2014[69]\end{array}$ & $\begin{array}{l}\text { https://github.com/biobakery/kneadda } \\
\text { ta }\end{array}$ \\
\hline & Bowtie2 & $\begin{array}{l}\text { Langmead et al., } \\
2012[43]\end{array}$ & $\begin{array}{l}\text { https://github.com/biobakery/kneadda } \\
\text { ta }\end{array}$ \\
\hline & FastQC & $\begin{array}{l}\text { Andrew, } 2017 \\
\text { [44] }\end{array}$ & $\begin{array}{l}\text { https://www.bioinformatics.babraham } \\
\text {.ac.uk/projects/fastqc/ }\end{array}$ \\
\hline $\begin{array}{l}\text { Taxonomy } \\
\text { assignment }\end{array}$ & Kraken2 & $\begin{array}{l}\text { Wood et al., } \\
2019[45]\end{array}$ & $\begin{array}{l}\text { https://github.com/DerrickWood/krak } \\
\text { en2/wiki\#downloads }\end{array}$ \\
\hline $\begin{array}{l}\text { Estimate relative } \\
\text { abundance of } \\
\text { species or genera }\end{array}$ & Bracken2 & $\begin{array}{l}\text { Lu et al., } 2017 \\
\text { [46] }\end{array}$ & $\begin{array}{l}\text { https://github.com/jenniferlu717/Brac } \\
\text { ken }\end{array}$ \\
\hline $\begin{array}{l}\text { Compute hash } \\
\text { sketches from } \\
\text { DNA sequence }\end{array}$ & Sourmash & $\begin{array}{l}\text { Brown et al., } \\
2016[51]\end{array}$ & $\begin{array}{l}\text { https://sourmash.readthedocs.io/en/lat } \\
\text { est/ }\end{array}$ \\
\hline \multirow[t]{2}{*}{$\begin{array}{l}\text { Replication of } \\
\text { bacterial species }\end{array}$} & GRiD & $\begin{array}{l}\text { Emiola et al., } \\
2018[68]\end{array}$ & https://github.com/ohlab/GRiD \\
\hline & Pathoscope 2.0 & $\begin{array}{l}\text { Hong et al., } 2014 \\
{[70]}\end{array}$ & $\begin{array}{l}\text { https://github.com/PathoScope/Patho } \\
\text { Scope }\end{array}$ \\
\hline $\mathbf{R}$ packages & & & \\
\hline
\end{tabular}




\begin{tabular}{|c|c|c|c|}
\hline $\begin{array}{l}\text { Data import, } \\
\text { filtering, and } \\
\text { processing }\end{array}$ & phyloseq & $\begin{array}{l}\text { McMurdie et al., } \\
2013 \text { [49] }\end{array}$ & $\begin{array}{l}\text { https://joey711.github.io/phyloseq/ind } \\
\text { ex.html }\end{array}$ \\
\hline \multirow[t]{2}{*}{$\begin{array}{l}\text { Core microbiota } \\
\text { analysis }\end{array}$} & DMwR & $\begin{array}{l}\text { Amunategui, } \\
2014[53]\end{array}$ & http://amunategui.github.io/smote/ \\
\hline & randomForest & $\begin{array}{l}\text { Breiman et al., } \\
2018[52]\end{array}$ & $\begin{array}{l}\text { https://cran.r- } \\
\text { project.org/web/packages/randomFor } \\
\text { est/index.html }\end{array}$ \\
\hline \multirow[t]{3}{*}{ Alpha-diversity } & phyloseq & $\begin{array}{l}\text { McMurdie et al., } \\
2013 \text { [49] }\end{array}$ & $\begin{array}{l}\text { https://joey711.github.io/phyloseq/ind } \\
\text { ex.html }\end{array}$ \\
\hline & microbiomeSeq & $\begin{array}{l}\text { Ssekagiri et al., } \\
2017[60]\end{array}$ & $\begin{array}{l}\text { https://github.com/umerijaz/microbiom } \\
\text { eSeq }\end{array}$ \\
\hline & rstatix & $\begin{array}{l}\text { Kassambara, } \\
2021[48]\end{array}$ & $\begin{array}{l}\text { https://cran.r- } \\
\text { project.org/web/packages/rstatix/inde } \\
\text { x.html }\end{array}$ \\
\hline Beta-diversity & phyloseq & $\begin{array}{l}\text { McMurdie et al., } \\
2013 \text { [49] }\end{array}$ & $\begin{array}{l}\text { https://joey711.github.io/phyloseq/ind } \\
\text { ex.html }\end{array}$ \\
\hline \multirow{2}{*}{$\begin{array}{l}\text { Differential } \\
\text { abundance } \\
\text { analysis }\end{array}$} & Maaslin2 & $\begin{array}{l}\text { Malick et al., } \\
2021[28]\end{array}$ & $\begin{array}{l}\text { https://huttenhower.sph.harvard.edu/maas } \\
\underline{\text { lin/ }}\end{array}$ \\
\hline & ANCOMBC & $\begin{array}{l}\text { Lin et al., } 2020 \\
\text { [67] }\end{array}$ & $\begin{array}{l}\text { http://www.bioconductor.org/package } \\
\text { s/release/bioc/vignettes/ANCOMBC/i } \\
\text { nst/doc/ANCOMBC.html }\end{array}$ \\
\hline
\end{tabular}




\begin{tabular}{|l|l|l|l|}
\hline ADONIS \& & vegan & Oksanen et al., & https://cran.r- \\
ANOSIM & & $2020[66]$ & project.org/web/packages/vegan/inde \\
& & & x.html \\
\hline
\end{tabular}

591 Ethics approval and consent to participate

592 This study was approved by the Medical Ethics Committee of Universiti Malaya Medical Centre

593 (UMMC) (Reference No.: 2017925-5593), National Medical Research Register (NMRR),

594 Ministry of Health, Malaysia (Reference No.: NMRR-17-3055-37252), New York University

595 (NYU) Institutional Review Board (IRB) (Reference No: i17-01068), the Department of Orang

596 Asli Development (JAKOA) [Reference No.: JAKOA/pp.30.052Jld13 (12) \&

597 JAKOA/pp.30.052Jld14 (47)] and the chiefs of respective villages (Tok Batin) before enrolling

598 the indigenous community in this study. An oral briefing using Malay language (the national

599 language for Malaysia) on the purpose and the procedure of this study were explained to all the

600 participants by the investigator. Written consent was attained from all adult participants aged 18

601 and above. For children under 18 years old, written parental consent was obtained from their

602 respective parents or guardian and assent form was obtained from children aged 7 to 17 years old.

603 Study exclusion criteria consisted of pregnant women, breastfeeding mothers and presence or 604 perceived presence of any clinically significant disease.

\section{Availability of data and material}

607 Raw data of gut metagenome has been deposited on the NCBI Sequence Read Archive (SRA) with 608 the BioProject No. PRJNA797994 and BioSample accession No. SAMN25042866-25043515. 


\section{Competing Interests}

610 Ken Cadwell has received research support from Pfizer, Takeda, Pacific Biosciences, Genentech,

611 and Abbvie. Ken Cadwell has consulted for or received honoraria from Puretech Health,

612 Genentech, and Abbvie. Ken Cadwell holds U.S. patent 10,722,600 and provisional patent

$61362 / 935,035$ and 63/157,225.

614 Other authors have declared no competing interests exist.

\section{Funding}

617 This research was supported in part by the Intramural Research Program of the NIH, National

618 Institute of Allergy and Infectious Diseases (NIAID) to P.L. and the NIAID 5R01AI130945 to

619 K.C., as well as the Fundamental Research Grant Scheme (FRGS), Ministry of Higher Education,

620 Malaysia [FP004-2017A] and University of Malaya Special Research Fund Assistance [BKS005-

$6212017]$ to Y. A. L. L. The research funders had no role in study design, data collection and analysis.

\section{Acknowledgements}

624 We gratefully acknowledge JAKOA at the Ministry of Rural and Regional Development, Kuala

625 Lumpur and the Village Chiefs for granting us permission to conduct this study and their

626 cooperation during the whole course of study. We also thank all the participants for their voluntary

627 participation and commitment in this study. Special thanks extended to the postgraduate and

628 undergraduate volunteers for their valuable assistance during the fieldtrips. Further funding was

629 provided by Faculty Scholar grant from Kenneth Rainin Foundation (K.C.) and Judith \& Stewart

630 Colton Center of Autoimmunity (K.C.). This work utilized the computational resources of the 
NIAID/NIH high performance computing (HPC) LOCUS cluster

632 (https://locus.niaid.nih.gov/userportal/login.php?redirect=userportal\%2Findex.php).

\section{Author contributions}

635 PL, YALL \& KC conceived and designed the study; SCL, MZT, YXE \& ILL organized and 636 coordinated field work; SCL, MZT, YXE, NJY \& AVE carried out and oversaw the collection of 637 samples from Malaysia; KSN provided clinical advice during sample collections; MZT \& YXE 638 performed the experiments; SCL, MZT \& YXE analyzed and interpretation of all experimental 639 data; JD, ZC, PS \& AA advised and assisted in gut metagenome analysis; RN provided advice on 640 mathematical analysis; CCMB, KHC \& SS reviewed and edited the paper; PL, SCL, MZT \& YXE 641 wrote the paper with input from all authors.

\section{References}

644 1. Cho I, Blaser MJ. The human microbiome: at the interface of health and disease. Nat Rev $645 \quad$ Genet. 2012;13(4):260-70.

646 2. Lynch SV, Pedersen O. The Human Intestinal Microbiome in Health and Disease. N Engl

647 J Med. 2016;375(24):2369-79.

648 3. Almeida A, Nayfach S, Boland M, Strozzi F, Beracochea M, Shi ZJ, et al. A unified catalog 649 of 204,938 reference genomes from the human gut microbiome. Nat Biotechnol. $650 \quad 2021 ; 39(1): 105-114$.

651 4. Blaser MJ. The Past and Future Biology of the Human Microbiome in an Age of 652 Extinctions. Cell. 2018;172(6):1173-77. 
653 5. Loke P, Lim YA. Helminths and the microbiota: parts of the hygiene hypothesis. Parasite $654 \quad$ Immunol. 2015;37(6):314-323.

655 6. Blaser MJ, Falkow S. What are the consequences of the disappearing human microbiota? $656 \quad$ Nat Rev Microbiol. 2009;7(12):887-94.

657 7. Ramanan D, Bowcutt R, Lee SC, Tang MS, Kurtz ZD, Ding Y, et al. Helminth infection 658 promotes colonization resistance via type 2 immunity. Science. 2016;352(6285):608-12.

659 8. Jenkins TP, Rathnayaka Y, Perera PK, Peachey LE, Nolan MJ, Krause L, et al. Infections by human gastrointestinal helminths are associated with changes in faecal microbiota

9. Jenkins TP, Formenti F, Castro C, Piubelli C, Perandin F, Buonfrate D, et al. A comprehensive analysis of the faecal microbiome and metabolome of Strongyloides stercoralis infected volunteers from a non-endemic area. Sci Rep. 2018;8(1):15651.

665 10. Rosa BA, Supali T, Gankpala L, Djuardi Y, Sartono E, Zhou Y, et al. Differential human gut microbiome assemblages during soil-transmitted helminth infections in Indonesia and Liberia. Microbiome. 2018;6(1):33.

668 11. Easton AV, Quiñones M, Vujkovic-Cvijin I, Oliveira RG, Kepha S, Odiere MR, et al. The Impact of Anthelmintic Treatment on Human Gut Microbiota Based on Cross-Sectional and Pre- and Postdeworming Comparisons in Western Kenya. mBio. 2019;10(2):e0051919.

672 12. Chen H, Mozzicafreddo M, Pierella E, Carletti V, Piersanti A, Ali SM, et al. Dissection of the gut microbiota in mothers and children with chronic Trichuris trichiura infection in Pemba Island, Tanzania. Parasit Vectors. 2021;14(1):62. 
675 13. Lee SC, Tang MS, Lim YA, Choy SH, Kurtz ZD, Cox LM, et al. Helminth Colonization

676 Is Associated with Increased Diversity of the Gut Microbiota. PLoS Negl Trop Dis.

$677 \quad 2014 ; 8(5): \mathrm{e} 2880$.

678 14. Cooper P, Walker AW, Reyes J, Chico M, Salter SJ, Vaca M, et al. Patent Human 679 Infections with the Whipworm, Trichuris trichiura, Are Not Associated with Alterations $680 \quad$ in the Faecal Microbiota. PLoS One. 2013;8(10):e76573.

681 15. Cantacessi C, Giacomin P, Croese J, Zakrzewski M, Sotillo J, McCann L, et al. Impact of 682 experimental hookworm infection on the human gut microbiota. J Infect Dis. 683 2014;210(9):1431-4.

684 16. Kay GL, Millard A, Sergeant MJ, Midzi N, Gwisai R, Mduluza T, et al. Differences in the 685 Faecal Microbiome in Schistosoma haematobium Infected Children vs. Uninfected Children. PLoS Negl Trop Dis. 2015;9(6):e0003861.

687 17. Martin I, Djuardi Y, Sartono E, Rosa BA, Supali T, Mitreva M, et al. Dynamic changes in human-gut microbiome in relation to a placebo-controlled anthelminthic trial in Indonesia. PLoS Negl Trop Dis. 2018;12(8):e0006620.

690 18. Schneeberger PHH, Coulibaly JT, Gueuning M, Moser W, Coburn B, Frey JE, et al. Offtarget effects of tribendimidine, tribendimidine plus ivermectin, tribendimidine plus oxantel-pamoate, and albendazole plus oxantel-pamoate on the human gut microbiota. Int

694 19. Jovel J, Patterson J, Wang W, Hotte N, O'Keefe S, Mitchel T, et al. Characterization of the 695 Gut Microbiome Using 16S or Shotgun Metagenomics. Front Microbiol. 2016;7:459. 
696 20. Ranjan R, Rani A, Metwally A, McGee HS, Perkins DL. Analysis of the microbiome:

697 Advantages of whole genome shotgun versus 16S amplicon sequencing. Biochem Biophys

$698 \quad$ Res Commun. 2016;469(4):967-77.

699 21. Bowers RM, Kyrpides NC, Stepanauskas R, Harmon-Smith M, Doud D, Reddy TBK, et 700 al. Minimum information about a single amplified genome (MISAG) and a metagenomeassembled genome (MIMAG) of bacteria and archaea. Nat Biotechnol. 2017;35(8):725-

22. Nayfach S, Shi ZJ, Seshadri R, Pollard KS, Kyrpides NC. New insights from uncultivated genomes of the global human gut microbiome. Nature. 2019;568(7753):505-510.

23. Pasolli E, Asnicar F, Manara S, Zolfo M, Karcher N, Armanini F, et al. Extensive Unexplored Human Microbiome Diversity Revealed by Over 150,000 Genomes from Metagenomes Spanning Age, Geography, and Lifestyle. Cell. 2019;176(3):649-662.e20.

24. Almeida A, Mitchell AL, Boland M, Forster SC, Gloor GB, Tarkowska A, et al. A new genomic blueprint of the human gut microbiota. Nature. 2019;568(7753):499-504.

25. Forster SC, Kumar N, Anonye BO, Almeida A, Viciani E, Stares MD, et al. A human gut bacterial genome and culture collection for improved metagenomic analyses. Nat Biotechnol. 2019;37(2):186-192.

713 26. Zou Y, Xue W, Luo G, Deng Z, Qin P, Guo R, et al. 1,520 reference genomes from cultivated human gut bacteria enable functional microbiome analyses. Nat Biotechnol.

716 27. Kim CY, Lee M, Yang S, Kim K, Yong D, Kim HR, et al. Human reference gut microbiome catalog including newly assembled genomes from under-represented Asian metagenomes. Genome Med. 2021;13(1):134. 
719 28. Mallick H, Rahnavard A, McIver LJ, Ma S, Zhang Y, Nguyen LH, et al. Multivariable

720 Association Discovery in Population-scale Meta-omics Studies. PLoS Comput Biol.

$721 \quad 2021 ; 17(11):$ e1009442.

722 29. Ajibola O, Rowan AD, Ogedengbe CO, Mshelia MB, Cabral DJ, Eze AA, et al. Urogenital 723 schistosomiasis is associated with signatures of microbiome dysbiosis in Nigerian $724 \quad$ adolescents. Sci Rep. 2019;9(1):829.

725 30. Rubel MA, Abbas A, Taylor LJ, Connell A, Tanes C, Bittinger K, et al. Lifestyle and the 726 presence of helminths is associated with gut microbiome composition in Cameroonians. 727 Genome Biol. 2020;21(1):122.

728 31. Durazzi F, Sala C, Castellani G, Manfreda G, Remondini D, De Cesare A. Comparison 729 between $16 \mathrm{~S}$ rRNA and shotgun sequencing data for the taxonomic characterization of the 730 gut microbiota. Sci Rep. 2021;11(1):3030.

731 32. Toro-Londono MA, Bedoya-Urrego K, Garcia-Montoya GM, Galvan-Diaz AL, Alzate JF. 732 Intestinal parasitic infection alters bacterial gut microbiota in children. PeerJ. 2019;7:e6200.

734 33. Huwe T, Prusty BK, Ray A, Lee S, Ravindran B, Michael E. Interactions between Parasitic 735 Infections and the Human Gut Microbiome in Odisha, India. Am J Trop Med Hyg. $736 \quad 2019 ; 100(6): 1486-1489$.

737 34. Lee SC, Tang MS, Easton AV, Devlin JC, Chua LL, Cho I, et al. Linking the effects of helminth infection, diet and the gut microbiota with human whole-blood signatures. PLoS Pathog. 2019;15(12):e1008066.

740 35. Fierer N, Lennon JT. The generation and maintenance of diversity in microbial communities. Am J Bot. 2011;98(3):439-48. 
742 36. Zhou D, Zhang H, Bai Z, Zhang A, Bai F, Luo X, et al. Exposure to soil, house dust and 743 decaying plants increases gut microbial diversity and decreases serum immunoglobulin E 744 levels in BALB/c mice. Environ Microbiol. 2016;18(5):1326-37.

745 37. Shyam Prasad G, Girisham S, Reddy SM. Microbial transformation of albendazole. Indian $746 \quad$ J Exp Biol. 2010;48(4):415-20.

747 38. Lanusse CE, Nare B, Gascon LH, Prichard RK. Metabolism of albendazole and 748 albendazole sulphoxide by ruminal and intestinal fluids of sheep and cattle. Xenobiotica.

39. Moser W, Schindler C, Keiser J. Efficacy of recommended drugs against soil transmitted helminths: systematic review and network meta-analysis. BMJ. 2017;358:j4307.

752 40. Adisakwattana P, Yoonuan T, Phuphisut O, Poodeepiyasawat A, Homsuwan N, Gordon CA, et al. Clinical helminthiases in Thailand border regions show elevated prevalence levels using qPCR diagnostics combined with traditional microscopic methods. Parasit Vectors. 2020;13(1):416.

756 41. Montresor A, Crompton DWT, Hall A, Bundy DAP, Savioli L. Guidelines for the evaluation of soil-transmitted helminthiasis and schistosomiasis at community level. World Health Organization: Geneva. 1998.

759 42. Chin YT, Lim YA, Chong CW, Teh CS, Yap IK, Lee SC, et al. Prevalence and risk factors Infect Dis Poverty. 2016;5(1):77.

762 43. Langmead B, Salzberg SL. Fast gapped-read alignment with Bowtie 2. Nat Methods. 2012;9(4):357-9.

764 44. Andrews S. FastQC: a quality control tool for high throughput sequence data. 2010. 
765 45. Wood DE, Lu J, Langmead B. Improved metagenomic analysis with Kraken 2. Genome 766 Biol. 2019;20(1):257.

767 46. Lu J, Breitwieser FP, Thielen P, Salzberg SL. Bracken: Estimating species abundance in 768 metagenomics data. PeerJ Comput Sci. 2017;3:e104.

769 47. Wickham, H. ggplot2. tidyverse 2021. Available from: https://ggplot2.tidyverse.org/.

770 48. Kassambara A. rstatix: Pipe-Friendly Framework for Basic Statistical Tests. r-project 771 2021. https://cran.r-project.org/web/packages/rstatix/index.html. Accessed 11 October

773 49. McMurdie PJ, Holmes S. phyloseq: An R Package for Reproducible Interactive Analysis and Graphics of Microbiome Census Data. PLoS One. 2013;8(4):e61217.

775 50. Kassambara. A. ggpubr: 'ggplot2' Based Publication Ready Plots. datanovia 2021. 776 https://rpkgs.datanovia.com/ggpubr/. Accessed 11 October 2021.

777 51. Brown CT, Izber L. sourmash: a library for MinHash sketching of DNA. J. Open Source 778 Softw. 2016;1(5):27.

779 52. Breiman L, Cutler A, Liaw A, Breiman MW. randomForest: Breiman and Cutler's Random 780 Forests for Classification and Regression. r-project 2018. https://cran.r$781 \quad$ project.org/web/packages/randomForest/index.html. Accessed on 11 October 2021.

782 53. Amunategui M. SMOTE - Supersampling Rare Events in R. Github 2014. 783 http://amunategui.github.io/smote/\#. Accessed on 18 October 2021.

784 54. Putri VM, Masjkur M, Suhaeni C. Performance of SMOTE in a random forest and naive 785 Bayes classifier for imbalanced Hepatitis-B vaccination status. J Phys Conf Ser. 2021:1863(012073):9. 
787 55. Brownlee J. Tune Machine Learning Algorithms in R (random forest case study). Machine Learning Mastery 2016. https://machinelearningmastery.com/tune-machine-learningalgorithms-in-r/. Accessed on 11 October 2021.

56. Douglas G. Random Forest Tutorial. LangilleLab/microbiome_helper. 2020. https://github.com/LangilleLab/microbiome_helper/wiki/Random-Forest-Tutorial. Accessed on 11 October 2021.

57. Humboldt AV, Bonpland A. Essay on the Geography of Plants. University of Chicago Press. 2010.

58. Shannon CE. A Mathematical Theory of Communication. Bell Syst Tech J. 1948;27:379_ $423,623-656$.

59. Simpson EH. Measurement of Diversity. Nature. 1949;163.

60. Ssekagiri A, Sloan WT, Ijaz UZ. microbiomeSeq: An R package for microbial community analysis in an environmental context. University of Glasgow : School of Engineering 2017. http://userweb.eng.gla.ac.uk/umer.ijaz/projects/microbiomeSeq_Tutorial.html. Accessed

802 61. Wilcoxon F. Individual Comparisons by Ranking Methods. Biometrics. 1945;1(6):80-83.

803 62. Spearman C. Demonstration of Formulæ for True Measurement of Correlation. Am J $804 \quad$ Psychol. 1907;18(2):161-169.

805 63. Boogaart KG, Tolosana-Delgado R, Bren M. compositions: Compositional Data Analysis. r-project 2021. https://cran.r-project.org/web/packages/compositions/index.html.

808 64. Anderson MJ. Distance-based tests for homogeneity of multivariate dispersions. 809 Biometrics. 2006;62(1):245-253. 
810 65. Clarke KR. Non-parametric multivariate analyses of changes in community structure.

$811 \quad$ Austral Ecol. 1993;18(1):117-143.

812 66. Oksanen J, Blanchet FG, Friendly M, Kindt R, Legendre P, McGlinn D, et al. vegan:

813 Community Ecology Package. r-project 2020. https://cran.r-

$814 \quad$ project.org/web/packages/vegan/index.html.

815 67. Lin H, Peddada SD. Analysis of compositions of microbiomes with bias correction. Nat $816 \quad$ Commun. 2020;11(1):3514.

817 68. Emiola A, Oh J. High throughput in situ metagenomic measurement of bacterial replication 818 at ultra-low sequencing coverage. Nat Commun. 2018;9(1):4956.

819 69. Bolger AM, Lohse M, Usadel B. Trimmomatic: a flexible trimmer for Illumina sequence 820 data. Bioinformatics. 2014;30(15):2114-20.

821 70. Hong C, Manimaran S, Shen Y, Perez-Rogers JF, Byrd AL, Castro-Nallar E, et al. 822 PathoScope 2.0: a complete computational framework for strain identification in 823 environmental or clinical sequencing samples. Microbiome. 2014;2:33. 